\title{
Hermitian Mindlin Plate Wavelet Finite Element Method for Load Identification
}

\author{
Xiaofeng Xue, Xuefeng Chen, Xingwu Zhang, Baijie Qiao, and Jia Geng \\ State Key Laboratory for Manufacturing System Engineering, School of Mechanical Engineering, Xian Jiaotong University, \\ Xian 710049, China \\ Correspondence should be addressed to Xuefeng Chen; chenxf@mail.xjtu.edu.cn
}

Received 11 November 2015; Revised 5 May 2016; Accepted 20 June 2016

Academic Editor: Nerio Tullini

Copyright (C) 2016 Xiaofeng Xue et al. This is an open access article distributed under the Creative Commons Attribution License, which permits unrestricted use, distribution, and reproduction in any medium, provided the original work is properly cited.

\begin{abstract}
A new Hermitian Mindlin plate wavelet element is proposed. The two-dimensional Hermitian cubic spline interpolation wavelet is substituted into finite element functions to construct frequency response function (FRF). It uses a system's FRF and response spectrums to calculate load spectrums and then derives loads in the time domain via the inverse fast Fourier transform. By simulating different excitation cases, Hermitian cubic spline wavelets on the interval (HCSWI) finite elements are used to reverse load identification in the Mindlin plate. The singular value decomposition (SVD) method is adopted to solve the ill-posed inverse problem. Compared with ANSYS results, HCSWI Mindlin plate element can accurately identify the applied load. Numerical results show that the algorithm of HCSWI Mindlin plate element is effective. The accuracy of HCSWI can be verified by comparing the FRF of HCSWI and ANSYS elements with the experiment data. The experiment proves that the load identification of HCSWI Mindlin plate is effective and precise by using the FRF and response spectrums to calculate the loads.
\end{abstract}

\section{Introduction}

For many practical engineering problems, such as structural strength analysis, health monitoring, fault diagnosis, and vibration isolation, the knowledge of the dynamic loads acting on the structure is always required and important [1]. The use of plate and shell in the industry becomes increasingly important [2]. As shear deformation Mindlin plate has a very wide range of application [3], load identification for Mindlin plate is very meaningful. However, it is difficult to directly measure the loads in a dynamic system, especially the interaction force in complex structures [4]. An alternative is to obtain these loads by the inverse force identification, which is an important topic in the identification of Mindlin plate under operating conditions. Therefore, load identification methods are necessary when the direct measurement is unfeasible [5]. The main idea of load identification is to use the measured structural response signals to determine the excitation load. However, it is an ill-posed inverse problem [6]. Different methods have been developed to make use of the response measurements of a structure to estimate the input excitations [4]. Frequency-domain method is one of the most commonly used load identification methods. Structure FRF can be constructed by numerical simulation. It uses a system's FRF and response spectrums to calculate load spectrums and then derives loads in the time domain via the inverse fast Fourier transform [7, 8]. Through the experiment, some scholars studied load identification using the method of FRF [9]. As for an ill-posed inverse problem, regularization methods such as the SVD, Chebyshev-Based Method, cubic B-spline-based method [10], and Tiknohov regularization are often used to transform it into a wellposed problem for great identification accuracy. However, the frequency-domain requires a long stationary time to calculate the inversion of the FRF matrix at each frequency [11-13]. It should be noted that the load position or distribution are usually assumed to be known in the load identification procedure [6]. Liu analyzed the truncated SVD to overcome ill-posed FRF matrix inversion [14].

Many vibroacoustic studies use finite element method (FEM) model to predict the dynamic behavior of structures. The aim is to reverse the load identification of the model by calculating sources from the measurements of their effects. Current commercial software and computing resources allow 
engineers to create numerical models that can be applied for more complex structures [15]. Zhao [16] presented the sliding loads identification based on the FEM and the strain measurement point selection procedure without information on their initial positions and magnitudes. Bahra [17] adopted the frame frequencies and mode shapes in a FEM model and solved the axial loads until the difference between measured and model frequencies is minimized, thus inferring the member axial loads. Lage [18] proposed a twostep loads identification method: the first step identifies the number of forces and their locations based on the response transmissibility concept; the second step reconstructs the load vector. Berry [19] presented the theoretical developments based on the virtual fields method and processed numerically simulated data to validate the identification algorithm.

However, for many complicated problems, traditional FEM has some disadvantages, such as low efficiency, insufficient accuracy, and slow convergence to correct solutions. Load identification, an ill-posed inverse problem, needs multiresolution analysis to improve the accuracy and reduce the computational work. Due to the property of multiresolution analysis, wavelet provides a natural mechanism for decomposing the solution into a set of coefficients. Wavelet functions can be viewed as interpolating functions, which are similar to those used in signal and image processing. Basu [20] maintained that the finite difference and Ritztype methods have been largely replaced by the FEM, the boundary element method, and the mesh-less method, and in the near future it might be the turn of the waveletbased numerical method. Because wavelet finite element has the advantages of reasonable interpolating functions configuration and higher precision. One can use fewer elements to solve the load identification. HCSWI elements embody a prominent advantage of improving precision by adding the appropriate wavelet functions [21, 22]. By using the modified one-dimensional HCSWI elements, a multiscale wavelet-based numerical method can solve load identification in rod and Timoshenko beam. Compared with traditional FEM and B-spline wavelet on interval (BSWI) [23-29] finite element, the method of HCSWI rod and beam elements has the advantage of higher precision. The tensor product of the modified Hermitian wavelets expanded at each coordinate is used to construct two-dimensional Mindlin plate Hermitian wavelet interpolation function. The load identification based on FRF is restricted as the result is inaccurate when the frequency content is close to certain resonance frequency of the structure [30]. Hermitian wavelet finite element with high precision can alleviate this problem effectively.

In the present study, an effective new wavelet numerical method based on two-dimensional Mindlin plate Hermitian wavelets interpolation function is proposed to construct the FRF and recover the loads. The paper is organized as follows. Section 2 addresses Hermitian Mindlin plate wavelet element. Section 3 describes load identification of HCSWI. Next, Section 4 details the numerical implementation and analysis. Section 5 presents the experimental verification. Concluding remarks are included in Section 6.

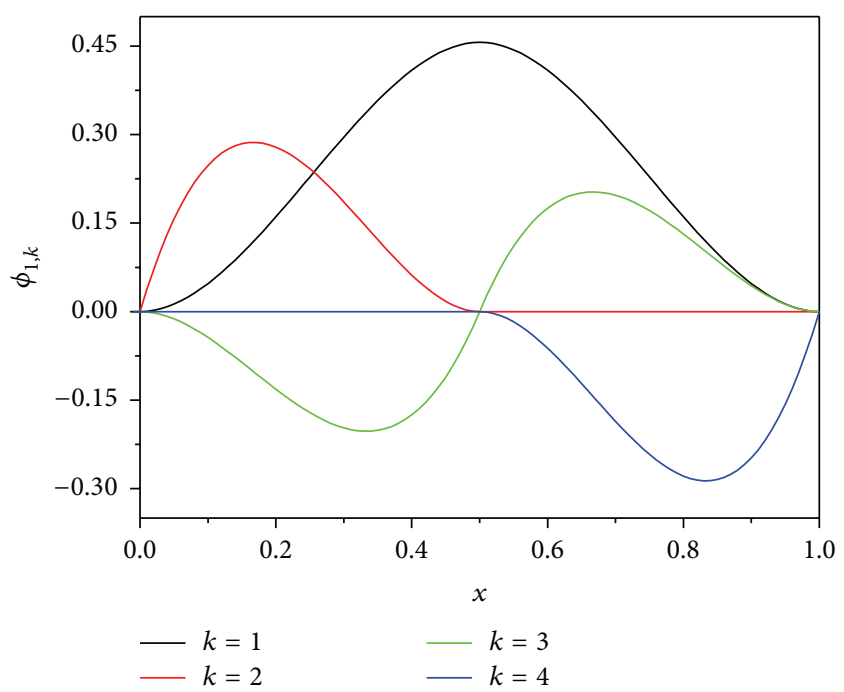

FIGURE 1: Scaling functions in scaling space $V_{1}$.

\section{Hermitian Mindlin Plate Wavelet Element}

A new Hermitian Mindlin plate wavelet element is constructed by the Hermitian cubic spline wavelets on interval interpolation functions. It can be substituted into finite element functions to solve the Hermitian wavelet stiffness and mass matrix for the purpose of obtaining FRF matrix. Through the inverse matrix of FRF matrix, one can solve the load identification. The method of SVD is adopted to solve the ill-posed inverse problem.

2.1. Hermitian Wavelet Interpolation Functions. Hermitian scaling functions $\phi_{1, k}$ are shown in Figure 1 and the equations are

$$
\begin{aligned}
& \phi_{1,1}(x):=\sqrt{\frac{5}{24}} \phi_{1}(2 x-1) \\
& \phi_{1,2}(x):=\sqrt{\frac{15}{4}} \phi_{2}(2 x) \\
& \phi_{1,3}(x):=\sqrt{\frac{15}{8}} \phi_{2}(2 x-1) \\
& \phi_{1,4}(x):=\sqrt{\frac{15}{4}} \phi_{2}(2 x-2),
\end{aligned}
$$

where

$$
\begin{gathered}
\phi_{1}(x):= \begin{cases}(x+1)^{2}(1-2 x) & \text { for } x \in[-1,0] \\
(1-x)^{2}(1+2 x) & \text { for } x \in[0,1] \\
0 & \text { for } x \notin[-1,1]\end{cases} \\
\phi_{2}(x):= \begin{cases}(x+1)^{2} x & \text { for } x \in[-1,0] \\
(x-1)^{2} x & \text { for } x \in[0,1] \\
0 & \text { for } x \notin[-1,1] .\end{cases}
\end{gathered}
$$


In order to solve the boundary problem of Hermitian interpolation wavelet, the modified Hermitian scaling functions with all the good performance of Hermitian wavelet [22] are proposed.

The modified Hermitian scaling functions $\phi_{1, k}^{m}$ are

$$
\begin{aligned}
\phi_{1,1}^{m}(x) & :=\sqrt{\frac{5}{24}} \phi_{1}(2 x) \\
\phi_{1,2}^{m}(x) & :=\sqrt{\frac{5}{24}} \phi_{1}(2 x-1) \\
\phi_{1,3}^{m}(x) & :=\sqrt{\frac{15}{4}} \phi_{2}(2 x) \\
\phi_{1,4}^{m}(x) & :=\sqrt{\frac{15}{8}} \phi_{2}(2 x-1) \\
\phi_{1,5}^{m}(x) & :=\sqrt{\frac{15}{4}} \phi_{2}(2 x-2) \\
\phi_{1,6}^{m}(x) & :=\sqrt{\frac{5}{24}} \phi_{1}(2 x-2)
\end{aligned}
$$

and the Hermitian wavelets $\psi_{j, k}$ are defined as

$$
\begin{aligned}
& \psi_{j, k}(x):=\frac{2^{-j / 2}}{\sqrt{729.6}} \psi_{1}\left(2^{j} x-\frac{k}{2}\right) \\
& \text { for } k=2,4, \ldots, 2^{j+1}-2 \\
& \psi_{j, k}(x):=\frac{2^{-j / 2}}{\sqrt{153.6}} \psi_{2}\left(2^{j} x-\frac{k-1}{2}\right) \\
& \quad \text { for } k=3,5, \ldots, 2^{j+1}-1 \\
& \psi_{j, 1}(x):=\frac{2^{-j / 2}}{\sqrt{76.8}} \psi_{2}\left(2^{j} x\right) \\
& \psi_{j, 2^{j+1}}(x):=\frac{2^{-j / 2}}{\sqrt{76.8}} \psi_{2}\left(2^{j} x-2^{j}\right),
\end{aligned}
$$

where

$$
\begin{aligned}
\psi_{1}(x)= & -2 \phi_{1}(2 x+1)+4 \phi_{1}(2 x)-2 \phi_{1}(2 x-1) \\
& -21 \phi_{2}(2 x+1)+21 \phi_{2}(2 x-1) \\
\psi_{2}(x)= & \phi_{1}(2 x+1)-\phi_{1}(2 x-1)+9 \phi_{2}(2 x+1) \\
& +12 \phi_{2}(2 x)+9 \phi_{2}(2 x-1) .
\end{aligned}
$$

All the modified Hermitian scaling functions $\phi_{1, k}^{m}$ and wavelets functions $\psi_{j, k}$ on the interval $[0,1]$ are shown in Figures 2 and 3, respectively. The special properties of Hermitian wavelet bases are

$$
\begin{aligned}
\left\langle\phi_{1, k}^{m \prime}, \psi_{j, k}^{\prime}\right\rangle & =\int_{0}^{1} \phi_{1, k}^{m \prime} \psi_{j, k}^{\prime} d x=0 \quad \forall j, k \\
\left\langle\psi_{j_{1}, k}^{\prime}, \psi_{j_{2}, k}^{\prime}\right\rangle & =\int_{0}^{1} \psi_{j_{1}, k}^{\prime} \psi_{j_{2}, k}^{\prime} d x=0
\end{aligned}
$$

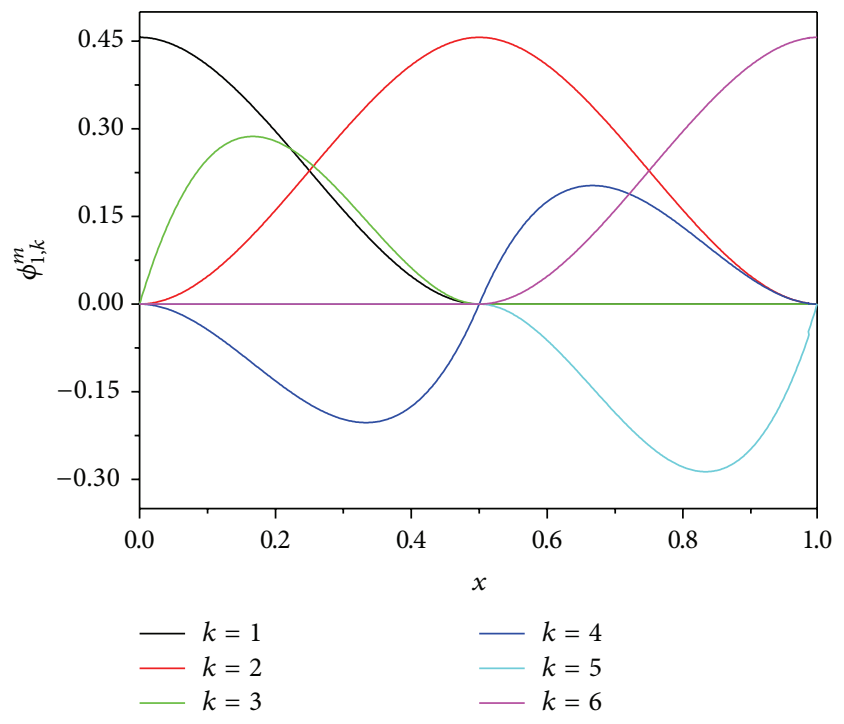

FIGURE 2: Modified scaling functions in scaling space $V_{1}$.

The wavelet bases in $V_{j}$ can be written by

$$
\boldsymbol{\Phi}_{j}=\left\{\varphi_{1}, \psi_{1}, \psi_{2}, \ldots, \psi_{j-1}\right\},
$$

where $\varphi_{1}=\left\{\phi_{1,1}^{m}, \phi_{1,2}^{m}, \phi_{1,3}^{m}, \phi_{1,4}^{m}, \phi_{1,5}^{m}, \phi_{1,6}^{m}\right\}$ denotes the modified Hermitian scaling functions in $V_{1}$ and $\psi_{s}(s=1,2, \ldots, j-$ 1 ) is the wavelet basis in $W_{s}$ (i.e., $\psi_{s}=\left\{\psi_{s, 1}, \psi_{s, 2}, \ldots, \psi_{s, 2^{s+1}}\right\}$ ).

The modified scaling functions $\phi_{1, k}^{m}$ and the corresponding wavelets $\psi_{j, k}$ in the multiresolution approximation space $\mathbf{H}_{0}^{1}(0,1)$ are constructed through one-dimensional multiresolution analysis of HCSWI on the interval $[0,1]$. A separable wavelet semiorthonormal basis at scale $j$ of $L_{2}(\mathbf{R})$ is constructed with tensor products of the multiresolution approximation space $\mathbf{H}_{0}^{1}(0,1) . V_{1}$ is the initial scaling space, and $W_{j}(j=1,2, \ldots)$ indicates the wavelet space at different levels. Therefore, the subspace of tensor product is spanned by $\boldsymbol{\varphi}_{j}=\boldsymbol{\Phi}_{j-1} \otimes \boldsymbol{\Phi}_{j-1}$. The scaling functions are $\boldsymbol{\varphi}_{1}=\varphi_{1} \otimes \varphi_{1}$; the appropriate wavelet functions are $\varphi_{2}=\Phi_{1} \otimes \Phi_{1}, \varphi_{3}=\Phi_{2} \otimes \Phi_{2}$. As Figure 4 shows, the tensor product of HCSWI scaling functions and wavelet functions are generated by the onedimensional HCSWI at scales $j=1,2$.

2.2. Mindlin Plate Theory. Hermitian wavelet interpolation function can be substituted into the potential function for Mindlin plate to solve the stiffness and mass matrix for load identification. According to Mindlin plate theory, the elemental generalized function of potential energy for Mindlin plate bending problem is [31]

$$
\begin{aligned}
\Pi_{p}= & \int_{\Omega_{e}} \frac{1}{2} \boldsymbol{\kappa}^{\mathrm{T}} \mathbf{G}_{\mathbf{D}} \boldsymbol{\kappa} d x d y-\int_{\Omega_{e}} \frac{1}{2} \boldsymbol{\gamma}^{\mathrm{T}} \mathbf{G}_{\mathbf{C}} \gamma d x d y \\
& -\int_{S_{\sigma}} q \mathbf{w} d x d y,
\end{aligned}
$$




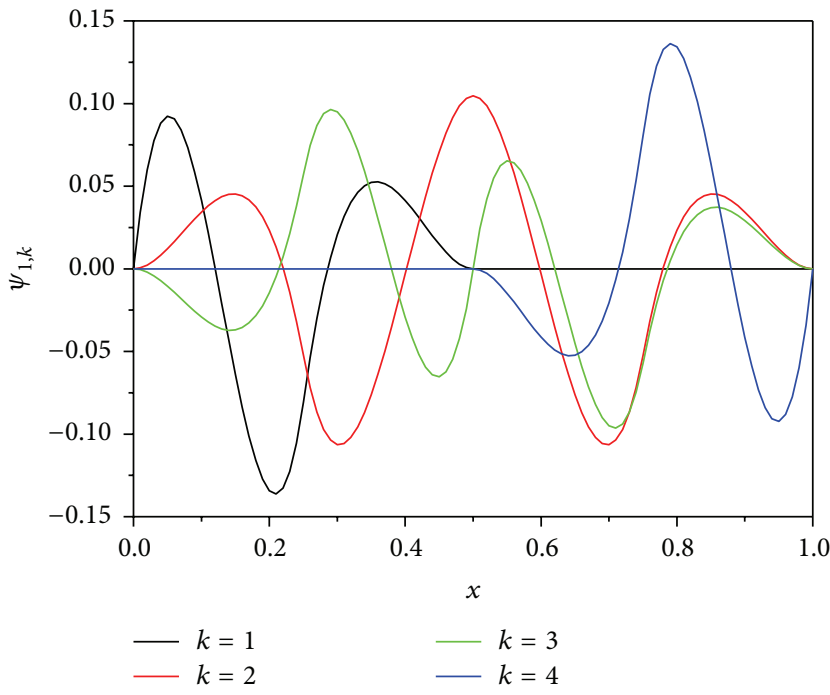

(a)

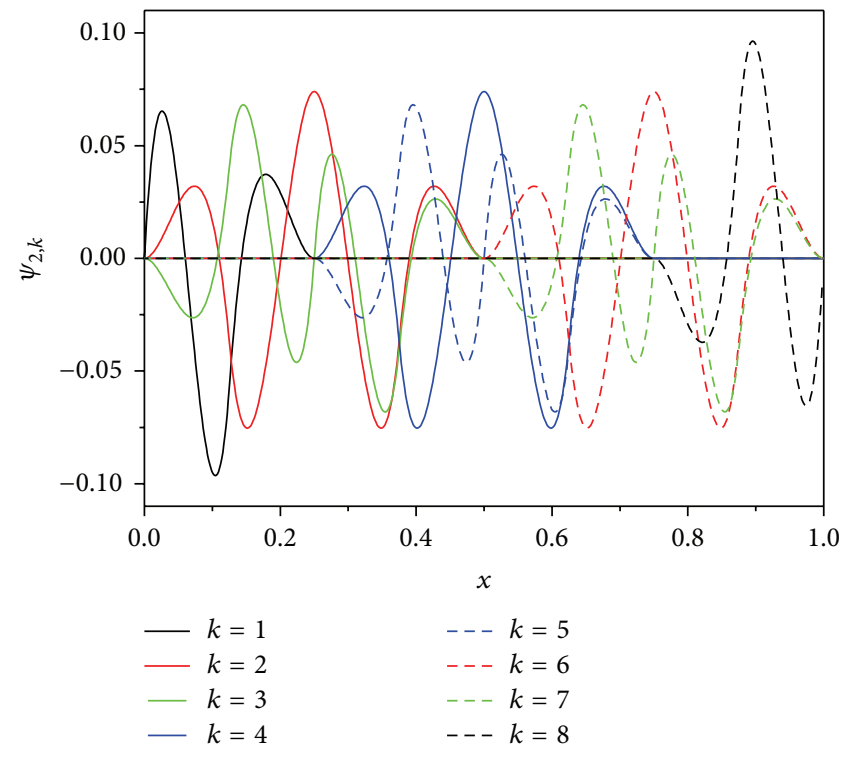

(b)

FIGURE 3: Wavelets in wavelet space $W$ : (a) wavelet in wavelet space $W_{1}$ and (b) wavelet in wavelet space $W_{2}$.



(a)

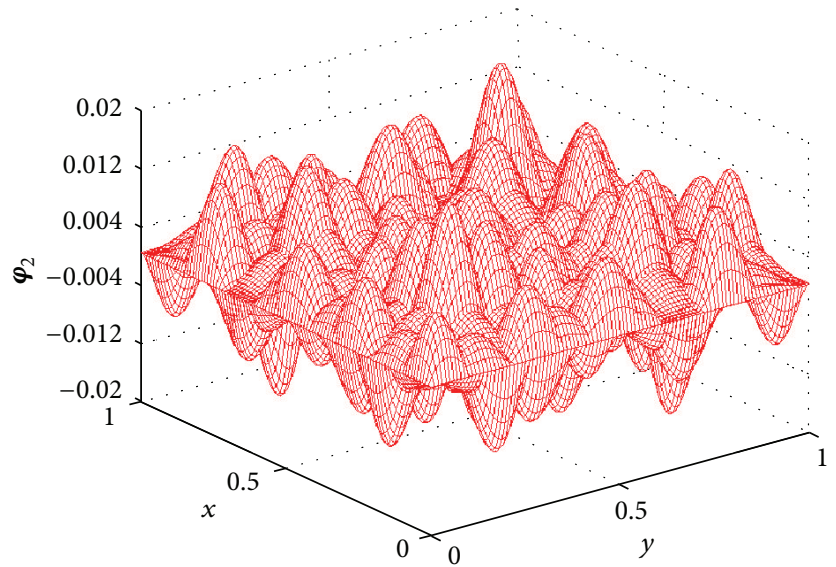

(b)

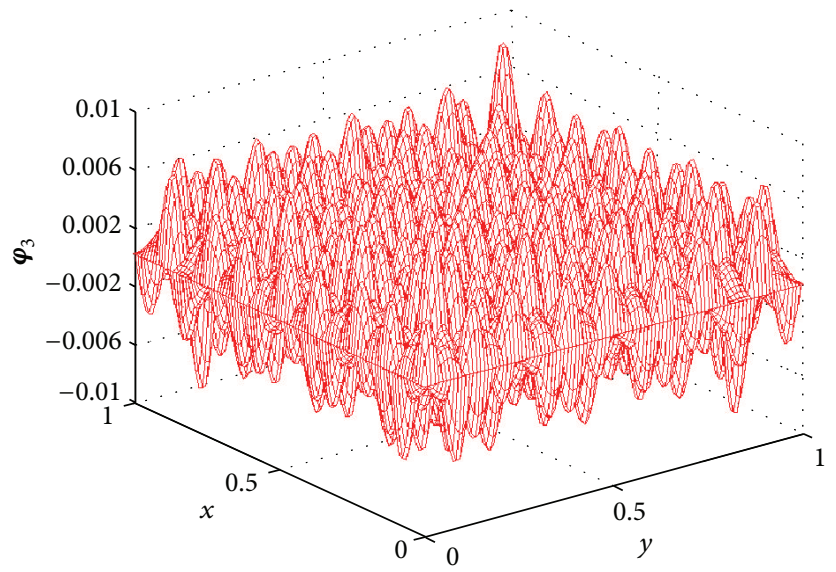

(c)

FIGURE 4: Tensor product HCSWI interpolation function: (a) modified scaling functions in scaling space $V_{1}$; (b) wavelet in wavelet space $W_{1}$; (c) wavelet in wavelet space $W_{2}$. 




FIGURE 5: Rectangle elemental solving domain in Mindlin plate.

where

$$
\begin{aligned}
\boldsymbol{\kappa} & =\left\{-\frac{\partial \theta_{x}}{\partial x}-\frac{\partial \theta_{y}}{\partial y}-\left(\frac{\partial \theta_{x}}{\partial y}+\frac{\partial \theta_{y}}{\partial x}\right)\right\}^{\mathrm{T}} \\
\boldsymbol{\gamma} & =\left\{\left(\frac{\partial w}{\partial x}-\theta_{x}\right)\left(\frac{\partial w}{\partial y}-\theta_{y}\right) 0\right\}^{\mathrm{T}} \\
\mathbf{G}_{\mathbf{D}} & =G_{d}\left[\begin{array}{llc}
1 & \mu & 0 \\
\mu & 1 & 0 \\
0 & 0 & \frac{(1-\mu)}{2}
\end{array}\right] \\
\mathbf{G}_{\mathbf{C}} & =G_{c}\left[\begin{array}{lll}
1 & 0 & 0 \\
0 & 1 & 0 \\
0 & 0 & 0
\end{array}\right]
\end{aligned}
$$

in which $G_{d}=E h^{3} / 12\left(1-\mu^{2}\right)$ and $G_{c}=5 E h / 12(1+\mu)$, where $E$ is Young's modulus, $h$ is the plate thickness, and $\mu$ is Poisson's ratio.

Substituting $\boldsymbol{\kappa}, \boldsymbol{\gamma}, \mathbf{G}_{\mathrm{D}}, \mathbf{G}_{\mathrm{C}}$ into (8), we can obtain

$$
\begin{aligned}
\Pi_{p} & =\frac{G_{d}}{2}\left\{\int \int _ { \Omega _ { e } } \left[\left(\frac{\partial \theta_{x}}{\partial x}\right)^{2}+2 \mu \frac{\partial \theta_{x}}{\partial x} \frac{\partial \theta_{y}}{\partial y}+\left(\frac{\partial \theta_{y}}{\partial y}\right)^{2}\right.\right. \\
& \left.\left.+\frac{1-\mu}{2}\left(\frac{\partial \theta_{x}}{\partial y}+\frac{\partial \theta_{y}}{\partial x}\right)^{2}\right] d x d y\right\} \\
& -\iint_{\Omega_{e}} q \mathbf{w} d x d y+\frac{G_{c}}{2} \iint_{\Omega_{e}}\left[\left(\frac{\partial w}{\partial x}-\theta_{x}\right)^{2}+\left(\frac{\partial w}{\partial y}\right.\right. \\
& \left.\left.-\theta_{y}\right)^{2}\right] d x d y .
\end{aligned}
$$

When we use the two-dimensional tensor product of HCSWI scaling functions for scale $j$ to construct element on the elemental solving domain $\Omega_{e}$ as shown in Figure 5, each node has three degrees of freedom (DOFs), that is, $\theta_{i x}, \theta_{i y}$, $w_{i}\left(i=1,2, \ldots,(n+1)^{2}\right)$; the displacement function in the standard elemental solving domain $\Omega_{s}$ can be replaced by

$$
\begin{array}{r}
w(\xi, \eta)=\boldsymbol{\varphi} \mathbf{T}^{e} \mathbf{w}^{e} \\
\theta_{x}(\xi, \eta)=\boldsymbol{\varphi} \mathbf{T}^{e} \boldsymbol{\theta}_{x}^{e} \\
\theta_{y}(\xi, \eta)=\boldsymbol{\varphi} \mathbf{T}^{e} \boldsymbol{\theta}_{y}^{e},
\end{array}
$$

where physical DOFs of the whole elemental nodes are

$$
\begin{aligned}
& \mathbf{w}^{e}=\left\{\begin{array}{lllll}
w_{1} & w_{2} & \cdots & w_{(n+1)^{2}-1} & w_{(n+1)^{2}}
\end{array}\right\}^{\mathrm{T}} \\
& \boldsymbol{\theta}_{x}^{e}=\left[\begin{array}{llllll}
\theta_{1 x} & \theta_{2 x} & \cdots & \theta_{\left[(n+1)^{2}-1\right] x} & \theta_{(n+1)^{2} x}
\end{array}\right\}^{\mathrm{T}} \\
& \boldsymbol{\theta}_{y}^{e}=\left[\begin{array}{lllll}
\theta_{1 y} & \theta_{2 y} & \cdots & \theta_{\left[(n+1)^{2}-1\right] y} & \theta_{(n+1)^{2} y}
\end{array}\right\}^{\mathrm{T}} .
\end{aligned}
$$

For (10), first mapping the elemental solving domain $\Omega_{e}$ to the standard elemental solving domain $\Omega_{s}$ and then substituting (11) into (10) and according to variation principle, let $\delta \Pi_{p}=0$; we can obtain the elemental FEM solution

$$
\left[\begin{array}{lll}
\mathbf{K}^{e, 1} & \mathbf{K}^{e, 2} & \mathbf{K}^{e, 3} \\
\mathbf{K}^{e, 4} & \mathbf{K}^{e, 5} & \mathbf{K}^{e, 6} \\
\mathbf{K}^{e, 7} & \mathbf{K}^{e, 8} & \mathbf{K}^{e, 9}
\end{array}\right]\left[\begin{array}{c}
\mathbf{w}^{e} \\
\boldsymbol{\theta}_{x}^{e} \\
\boldsymbol{\theta}_{y}^{e}
\end{array}\right]=\left[\begin{array}{c}
\mathbf{F}^{e} \\
0 \\
0
\end{array}\right],
$$

where

$$
\begin{aligned}
& \mathbf{K}^{e, 1}=G_{d}\left\{\boldsymbol{\Gamma}_{1}^{1,1} \otimes \boldsymbol{\Gamma}_{2}^{0,0}+\frac{(1-\mu)}{2 \boldsymbol{\Gamma}_{1}^{0,0} \otimes \boldsymbol{\Gamma}_{2}^{1,1}}\right\}+G_{c} \boldsymbol{\Gamma}_{1}^{0,0} \otimes \boldsymbol{\Gamma}_{2}^{0,0} \\
& \mathbf{K}^{e, 2}=G_{d}\left\{\mu \boldsymbol{\Gamma}_{1}^{1,0} \otimes \boldsymbol{\Gamma}_{2}^{0,1}+\frac{(1-\mu)}{2 \boldsymbol{\Gamma}_{1}^{0,1} \otimes \boldsymbol{\Gamma}_{2}^{1,0}}\right\} \\
& \mathbf{K}^{e, 3}=-G_{c} \boldsymbol{\Gamma}_{1}^{0,1} \otimes \boldsymbol{\Gamma}_{2}^{0,0} \\
& \mathbf{K}^{e, 4}=\left(\mathbf{K}^{e, 2}\right)^{\mathrm{T}} \\
& \mathbf{K}^{e, 5}=G_{d}\left\{\boldsymbol{\Gamma}_{1}^{0,0} \otimes \boldsymbol{\Gamma}_{2}^{1,1}+\frac{(1-\mu)}{2 \boldsymbol{\Gamma}_{1}^{1,1} \otimes \boldsymbol{\Gamma}_{2}^{0,0}}\right\}+G_{c} \boldsymbol{\Gamma}_{1}^{0,0} \otimes \boldsymbol{\Gamma}_{2}^{0,0} \\
& \mathbf{K}^{e, 6}=-G_{c} \boldsymbol{\Gamma}_{1}^{0,0} \otimes \boldsymbol{\Gamma}_{2}^{0,1} \\
& \mathbf{K}^{e, 7}=\left(\mathbf{K}^{e, 3}\right)^{\mathrm{T}} \\
& \mathbf{K}^{e, 8}=\left(\mathbf{K}^{e, 6}\right)^{\mathrm{T}} \\
& \mathbf{K}^{e, 9}=G_{c}\left\{\boldsymbol{\Gamma}_{1}^{1,1} \otimes \boldsymbol{\Gamma}_{2}^{0,0}+\boldsymbol{\Gamma}_{1}^{0,0} \otimes \boldsymbol{\Gamma}_{2}^{1,1}\right\} .
\end{aligned}
$$

Due to the independent interpolation of $\theta_{x}, \theta_{y}$, and $w$, we can change the location of matrix element of the elemental FEM in solving (13) to get 


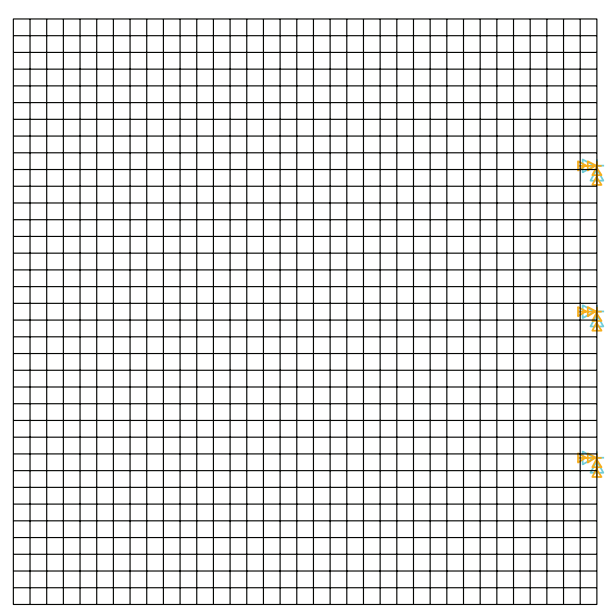

Figure 6: The $35 \times 35$ mesh of the Mindlin plate.
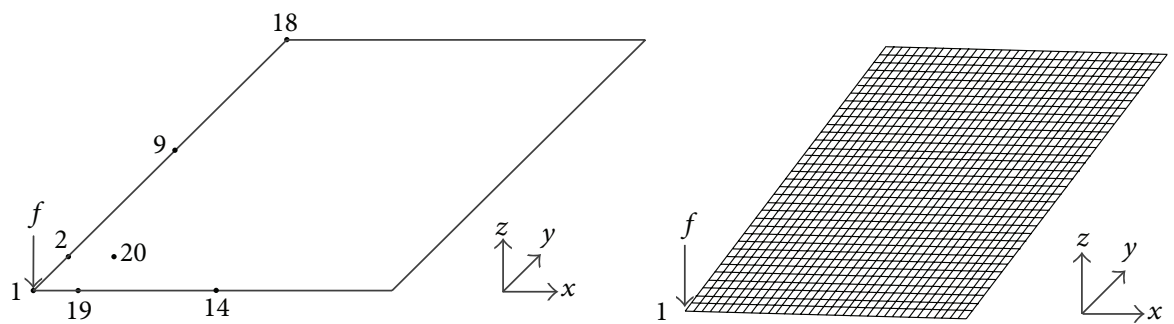

Figure 7: The location of $f$ in the Mindlin plate.

where

$$
\mathbf{K}_{i, j}^{e}=\left[\begin{array}{lll}
k_{i, j}^{e, 1} & k_{i, j}^{e, 2} & k_{i, j}^{e, 3} \\
k_{i, j}^{e, 4} & k_{i, j}^{e, 5} & k_{i, j}^{e, 6} \\
k_{i, j}^{e, 7} & k_{i, j}^{e, 8} & k_{i, j}^{e, 9}
\end{array}\right] \quad i, j=1,2, \ldots,(n+1)^{2} .
$$

$M$ is the consistent mass matrix as follows:

$$
\begin{aligned}
M= & \rho A h\left(T^{e}\right)^{\mathrm{T}}\left(\frac{d\left(\Gamma_{1}^{0,0} \otimes \Gamma_{2}^{0,0}\right)^{\mathrm{T}}}{d \xi}\right) \\
& \cdot\left(\frac{d\left(\Gamma_{1}^{0,0} \otimes \Gamma_{2}^{0,0}\right)}{d \xi}\right) T^{e},
\end{aligned}
$$

where $\rho$ is the density and $A$ is the area of the Mindlin plate.
In order to satisfy the continuity and compatibility at the interface between neighboring elements and the boundary condition, the stiffness matrix for dynamic analysis should be transformed into physical space. The corresponding elemental DOFs can be solved from wavelet coefficients into unknown field functions. The one-dimensional transformation matrices $T^{1}$ and $T^{2}$ can construct a twodimensional transformation matrix $T^{e}=T^{1} \otimes T^{2}$. The other procedures can follow those of the traditional FEM [32].

The first-order derivative of HCSWI has the property of continuity, which is suitable for the analysis of plate and shell structure. Therefore, Hermitian wavelet with high precision as a finite element interpolation function can be used in the Mindlin plate. 


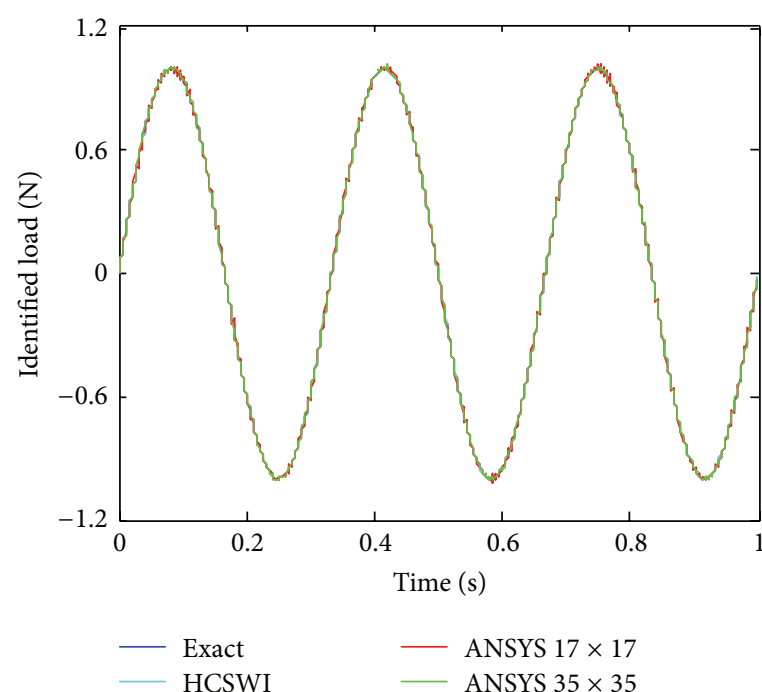

(a) Identified load $f$ at node 2

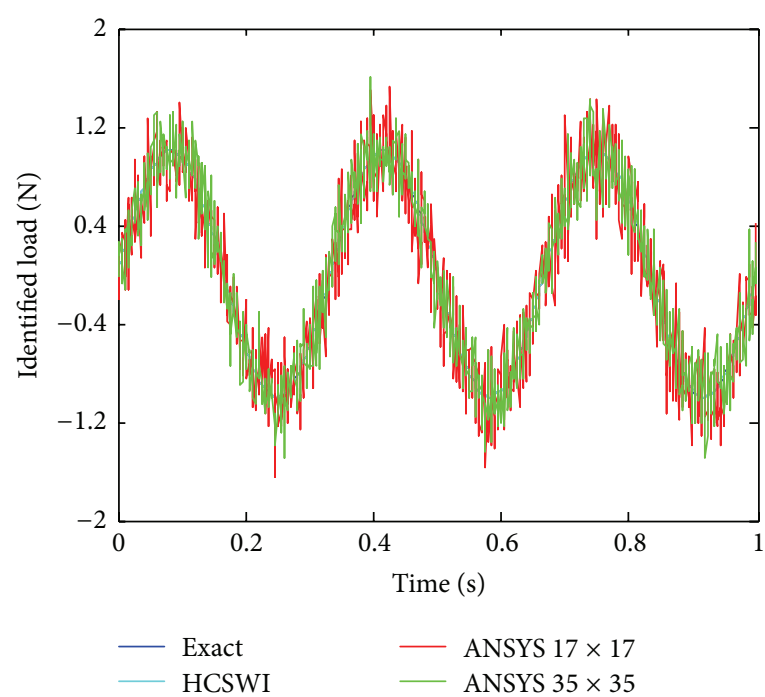

(c) Identified load $f$ at node 18

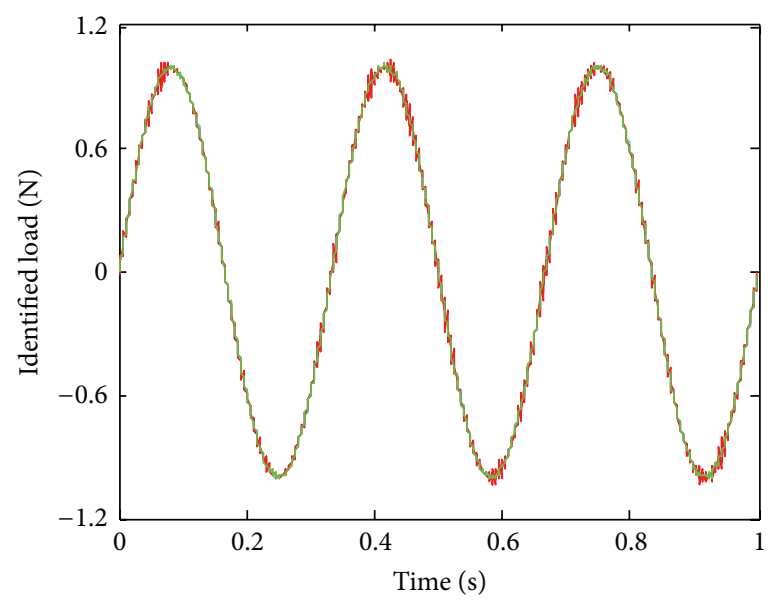

$\begin{array}{ll}\text { Exact } & - \text { ANSYS } 17 \times 17 \\ \text { HCSWI } & \text { ANSYS } 35 \times 35\end{array}$

(e) Identified load $f$ at node 20

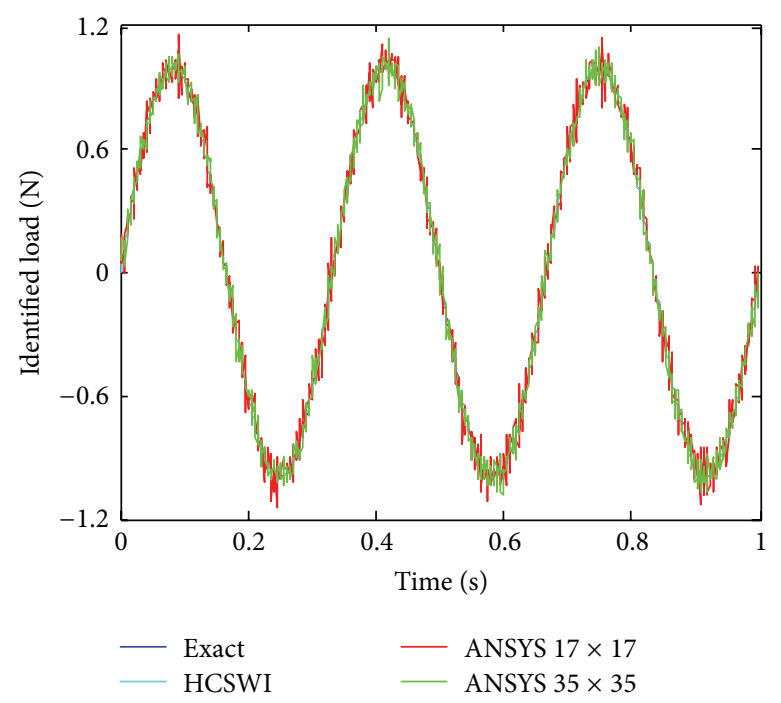

(b) Identified load $f$ at node 9

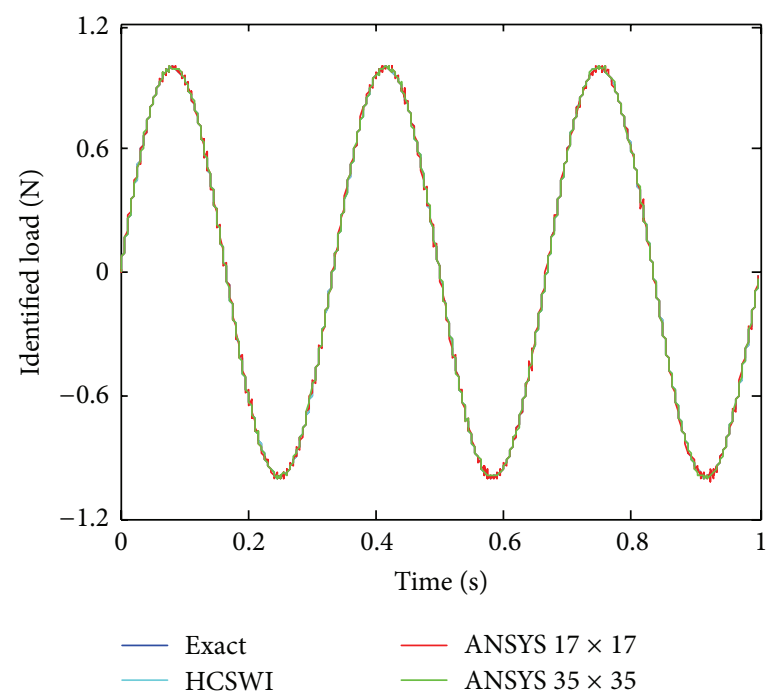

(d) Identified load $f$ at node 19



(f) Identified load $f$ at node 145

FIGURE 8: Load identification at different nodes with excitation $f$ applied at node 1. 

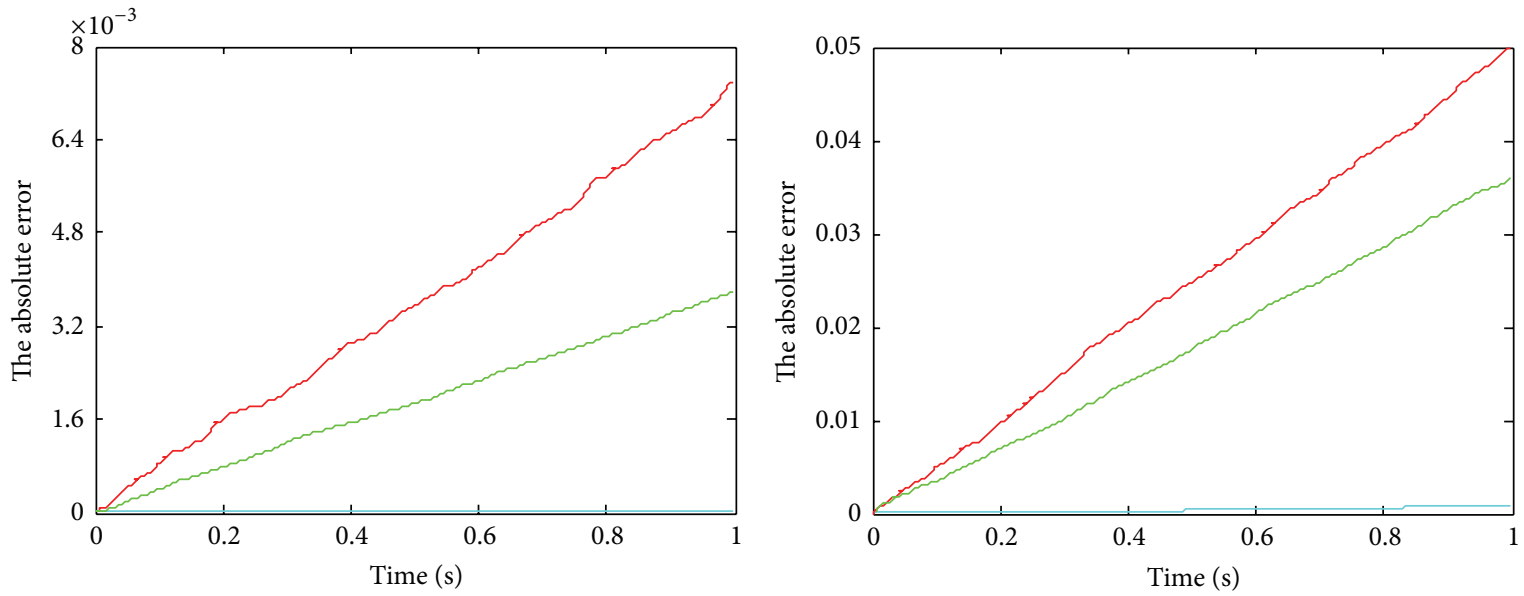

HCSWI
ANSYS $17 \times 17 \quad$ ANSYS $35 \times 35$

(a) Identified load $f$ at node 2

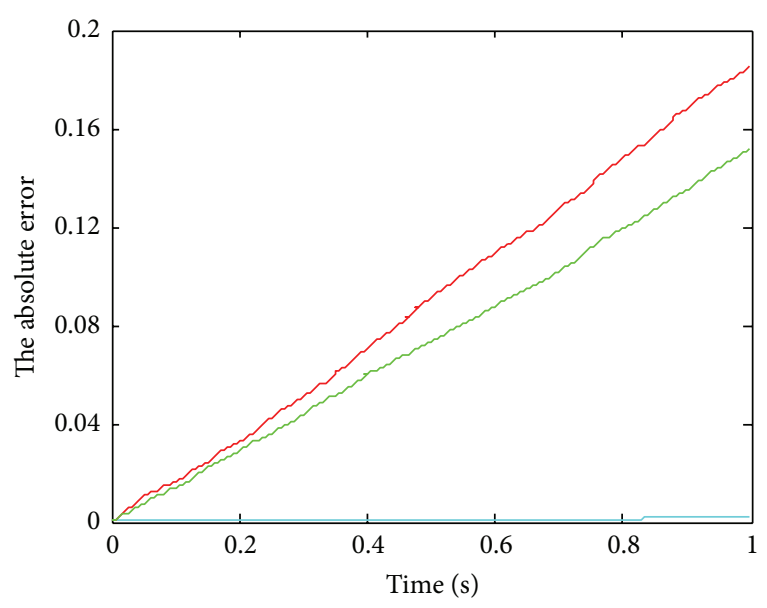

- HCSWI - ANSYS $35 \times 35$

ANSYS $17 \times 17$

(c) Identified load $f$ at node 18



(e) Identified load $f$ at node 20

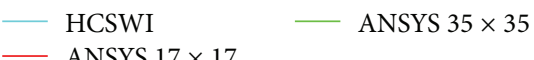

(b) Identified load $f$ at node 9

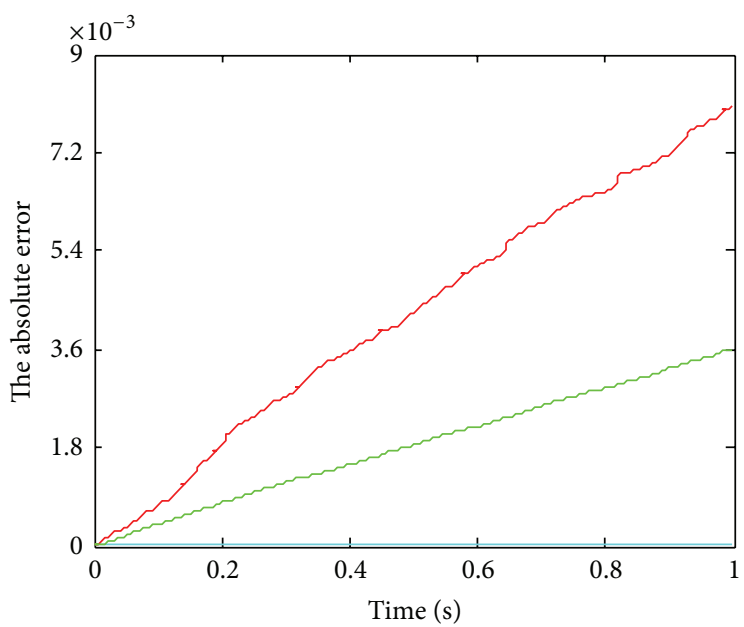

- HCSWI - ANSYS $35 \times 35$

(d) Identified load $f$ at node 19

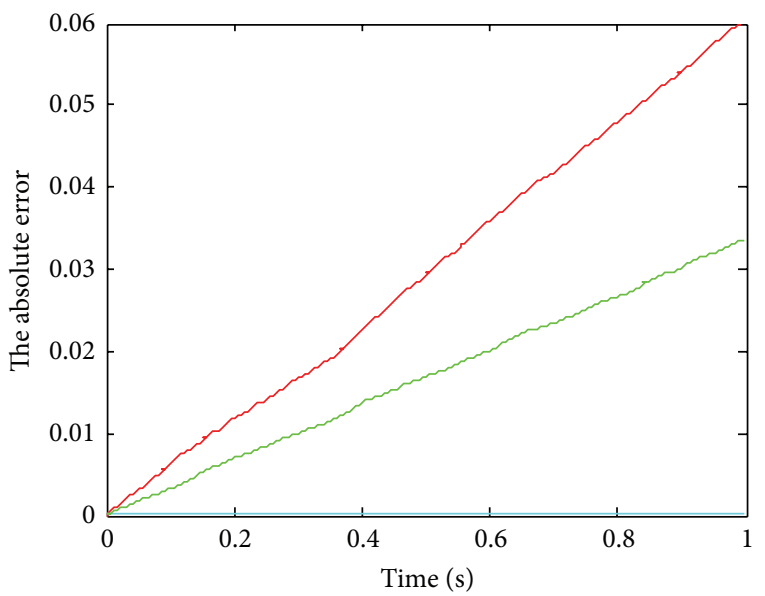

- HCSWI - ANSYS $35 \times 35$

- ANSYS $17 \times 17$

(f) Identified load $f$ at node 145

FIgURE 9: The absolute error with excitation $f$ applied at node 1 . 




HCSWI

ANSYS $35 \times 35$

- ANSYS $17 \times 17$

(a) Identified load $f$ at node 2
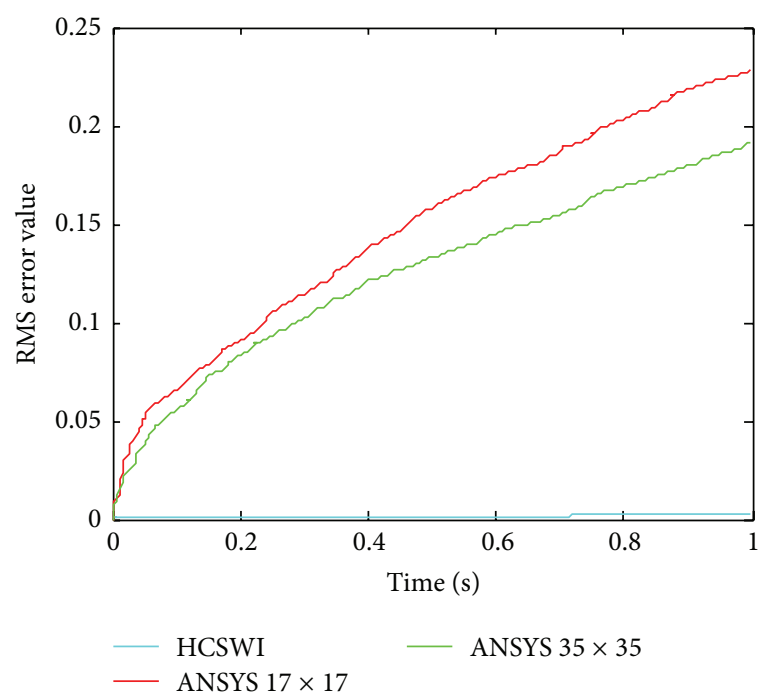

(c) Identified load $f$ at node 18



HCSWI

- ANSYS $35 \times 35$

- ANSYS $17 \times 17$

(e) Identified load $f$ at node 20

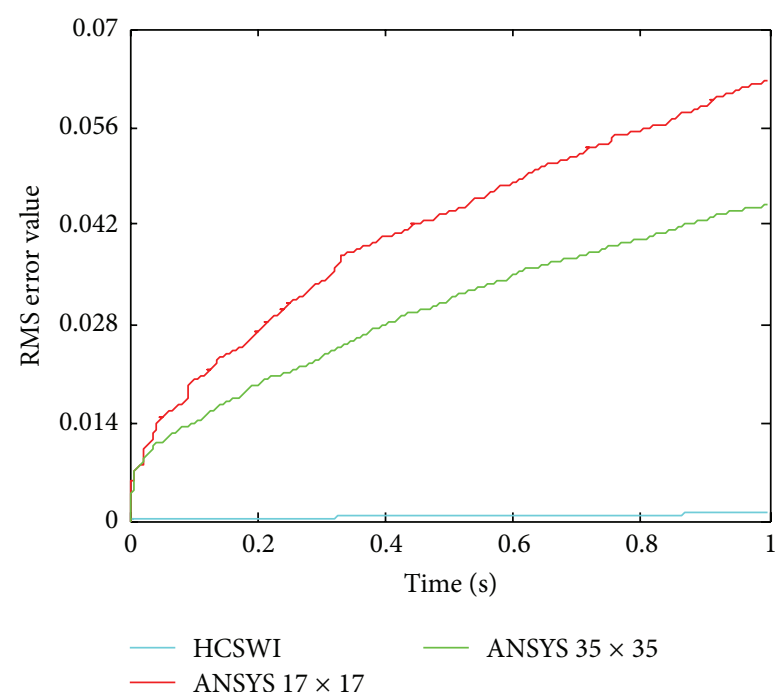

(b) Identified load $f$ at node 9



(d) Identified load $f$ at node 19

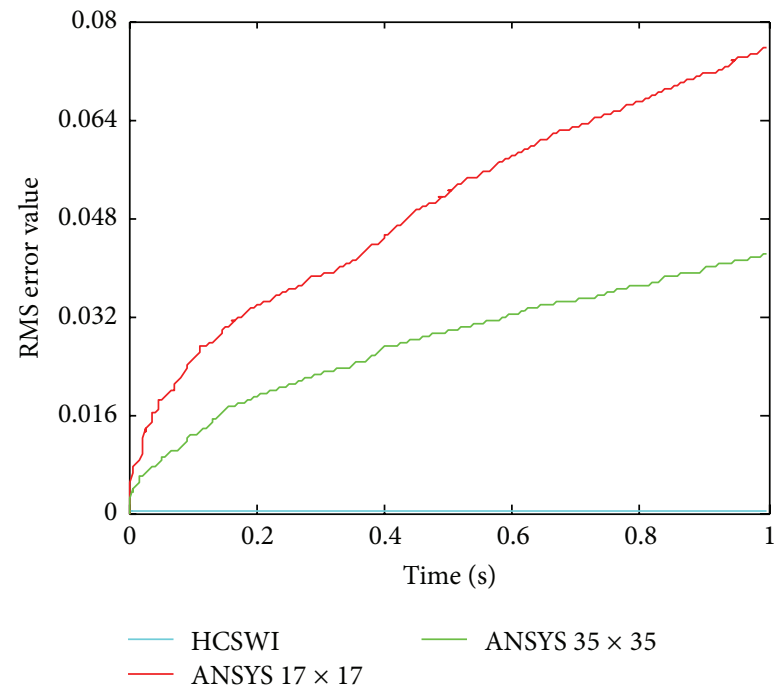

(f) Identified load $f$ at node 145

Figure 10: The root mean square error value with the excitation $f$ applied at node 1 . 


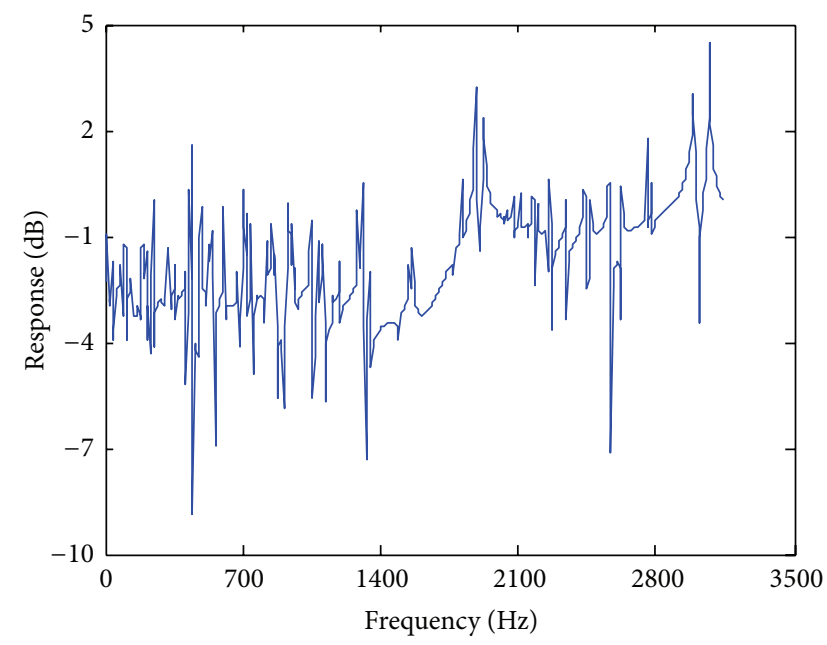

(a) Node 2



(c) Node 18



(e) Node 20



(b) Node 9



(d) Node 19



(f) Node 145

Figure 11: FRF with excitation $f$ applied at node 1. 


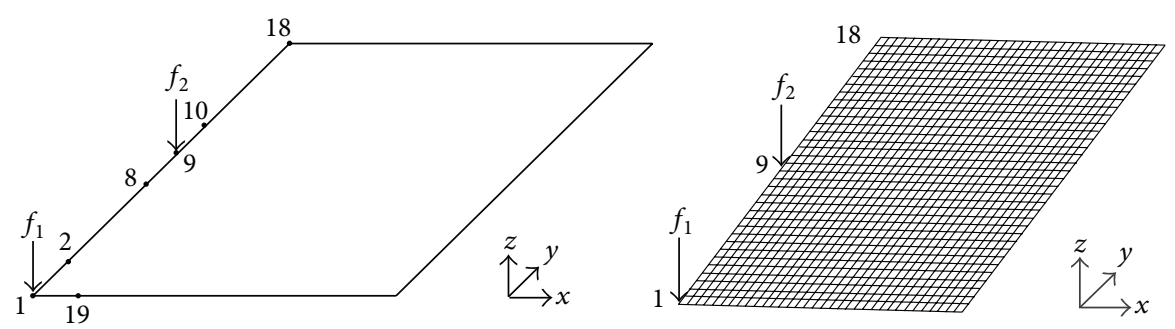

Figure 12: The location of $f_{1}$ and $f_{2}$ in the Mindlin plate.

2.3. Integral Solution about Mindlin Plate Stiffness Matrix. Hermitian interpolation function wavelet can be substituted into the potential function for Mindlin plate to solve the stiffness matrix; the integral solution equation is as follows:

$$
\Gamma_{1}^{0,0}=l_{e x} \Gamma^{0,0} .
$$

The key to realize the multiscale solution of mechanical structures is to compute the integral function.

For the integral $\Gamma^{0,0}=\int_{0}^{1} \boldsymbol{\Phi}^{\mathrm{T}} \boldsymbol{\Phi} d \xi$, the multiscale constant matrix is

$$
\Gamma^{0,0}=\left[\begin{array}{cccc}
\mathbf{D}_{\varphi_{1}, \varphi_{1}} & \mathbf{D}_{\varphi_{1}, \psi_{1}} & \cdots & \mathbf{D}_{\varphi_{1}, \psi_{j-1}} \\
& \mathbf{D}_{\psi_{1}, \psi_{1}} & \cdots & \mathbf{D}_{\psi_{1}, \psi_{j-1}} \\
& & \ddots & \vdots \\
\operatorname{sym} & & & \mathbf{D}_{\psi_{j-1}, \psi_{j-1}}
\end{array}\right],
$$

where the submatrix of $\Gamma^{0,0}$ can be calculated by

$$
\mathbf{D}_{x, y}=\int_{0}^{1} x^{\mathrm{T}} y d \xi, \quad x, y=\varphi_{1}, \psi_{1}, \ldots, \psi_{j-1} .
$$

Equation (20) reveals that only the submatrices $\mathbf{D}_{x, y}$, $\left(x=\psi_{1}, \ldots, \psi_{j-1} ; y=\psi_{l}\right)$ will be calculated while other submatrices remain the same when scale $l$ is lifted to $l+1$, thus increasing the calculating efficiency.

The integral solution equation is as follows:

$$
\Gamma_{1}^{0,1}=\Gamma^{0,1} .
$$

For the integral $\Gamma^{0,1}=\int_{0}^{1} \boldsymbol{\Phi}\left(d \boldsymbol{\Phi}^{\mathrm{T}} / d \xi\right) d \xi$, the multiscale constant matrix is

$$
\Gamma^{0,1}=\left[\begin{array}{cccc}
\mathbf{C}_{\varphi_{1}, \varphi_{1}} & \mathbf{C}_{\varphi_{1}, \psi_{1}} & \cdots & \mathbf{C}_{\varphi_{1}, \psi_{j-1}} \\
& \mathbf{C}_{\psi_{1}, \psi_{1}} & \cdots & \mathbf{C}_{\psi_{1}, \psi_{j-1}} \\
& & \ddots & \vdots \\
\text { An-sym } & & & \mathbf{C}_{\psi_{j-1}, \psi_{j-1}}
\end{array}\right],
$$

where the submatrix of $\Gamma^{0,1}$ can be calculated by

$\mathbf{C}_{x, y}=\int_{0}^{1} x \frac{d y^{\mathrm{T}}}{d \xi} d \xi, \quad x, y=\varphi_{1}, \psi_{1}, \ldots, \psi_{j-1}$.
Equation (23) reveals that only the submatrices $\mathbf{C}_{x, y},\left(x=\psi_{1}, \ldots, \psi_{j-1} ; y=\psi_{l}\right)$ will be calculated while other submatrices remain the same when scale $l$ is lifted to $l+1$, thus increasing the calculating efficiency.

The integral solution equation is as follows:

$$
\Gamma_{1}^{1,0}=l_{e x} \Gamma^{1,0}
$$

For the integral $\Gamma^{1,0}=\int_{0}^{1}\left(d \Phi^{\mathrm{T}} / d \xi\right) \Phi d \xi$, the multiscale constant matrix is

$$
\Gamma^{1,0}=\left[\begin{array}{cccc}
\mathbf{B}_{\varphi_{1}, \varphi_{1}} & \mathbf{B}_{\varphi_{1}, \psi_{1}} & \cdots & \mathbf{B}_{\varphi_{1}, \psi_{j-1}} \\
& \mathbf{B}_{\psi_{1}, \psi_{1}} & \cdots & \mathbf{B}_{\psi_{1}, \psi_{j-1}} \\
& & \ddots & \vdots \\
\text { An-sym } & & & \mathbf{B}_{\psi_{j-1}, \psi_{j-1}}
\end{array}\right],
$$

where the submatrix of $\Gamma^{1,0}$ can be calculated by

$\mathbf{B}_{x, y}=\int_{0}^{1} \frac{d x^{\mathrm{T}}}{d \xi} y d \xi, \quad x, y=\varphi_{1}, \psi_{1}, \ldots, \psi_{j-1}$

Equation (26) reveals that only the submatrices $\mathbf{B}_{x, y}$, $\left(x=\psi_{1}, \ldots, \psi_{j-1} ; y=\psi_{l}\right)$ can be calculated while other submatrices remain the same when scale $l$ is lifted to $l+1$, thus increasing the calculating efficiency.

The integral solution equation is as follows:

$$
\Gamma_{1}^{1,1}=\left(\frac{1}{l_{e x}}\right) \Gamma^{1,1}
$$

For the integral $\Gamma^{1,1}=\int_{0}^{1}\left(d \Phi^{\mathrm{T}} / d \xi\right)(d \Phi / d \xi) d \xi$, the multiscale constant matrix is

$$
\Gamma^{1,1}=\left[\begin{array}{cccc}
\mathbf{A}_{\varphi_{1}, \varphi_{1}} & \mathbf{A}_{\varphi_{1}, \psi_{1}} & \cdots & \mathbf{A}_{\varphi_{1}, \psi_{j-1}} \\
& \mathbf{A}_{\psi_{1}, \psi_{1}} & \cdots & \mathbf{A}_{\psi_{1}, \psi_{j-1}} \\
& & \ddots & \vdots \\
\operatorname{sym} & & & \mathbf{A}_{\psi_{j-1}, \psi_{j-1}}
\end{array}\right],
$$





(a1) Identified load $f_{1}$ at node 2

(a2) Identified load $f_{2}$ at node 2


$\begin{array}{ll}\text { Exact } & \text { ANSYS } 17 \times 17 \\ \text { HCSWI } & \text { ANSYS } 35 \times 35\end{array}$

(b1) Identified load $f_{1}$ at node 10

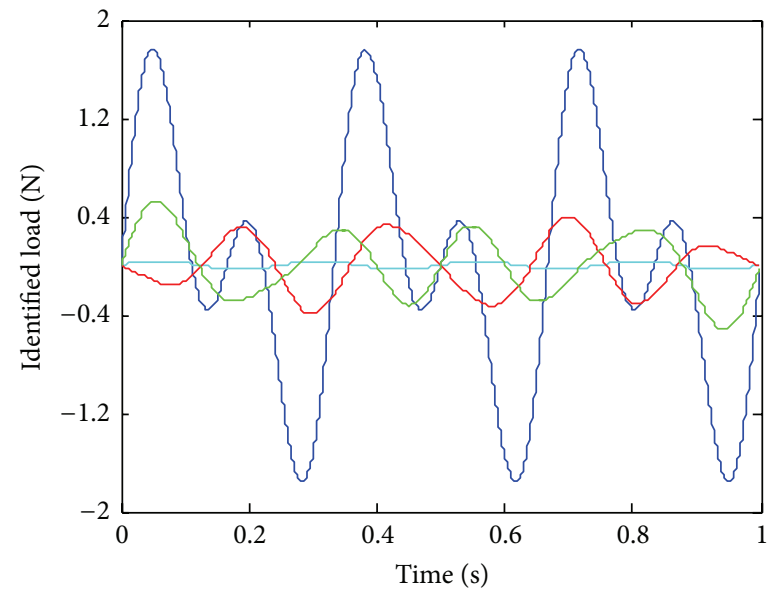

$\begin{array}{ll}- \text { Exact } & \text { ANSYS } 17 \times 17 \\ \text { HCSWI } & \text { ANSYS } 35 \times 35\end{array}$

(c1) Identified load $f_{1}$ at node 18



(b2) Identified load $f_{2}$ at node 10

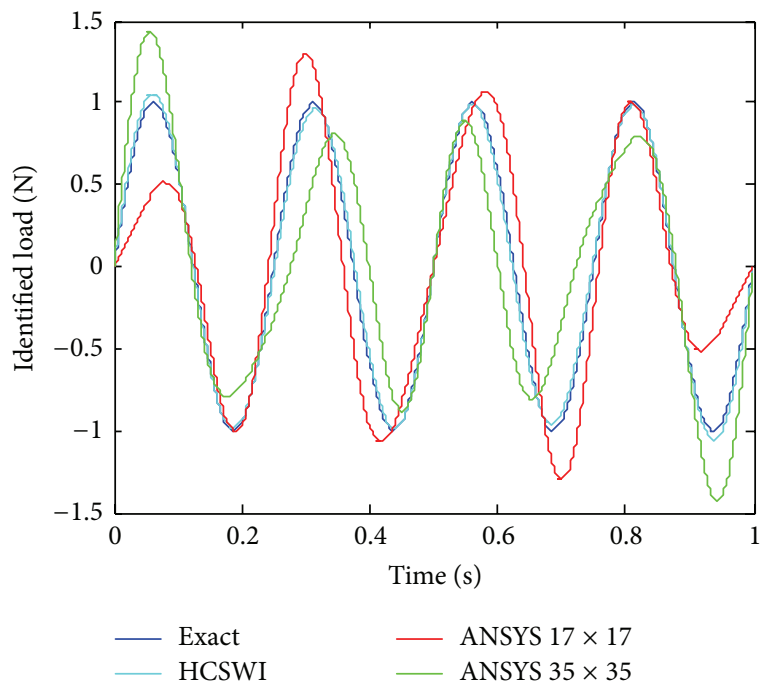

(c2) Identified load $f_{2}$ at node 18

Figure 13: Continued. 


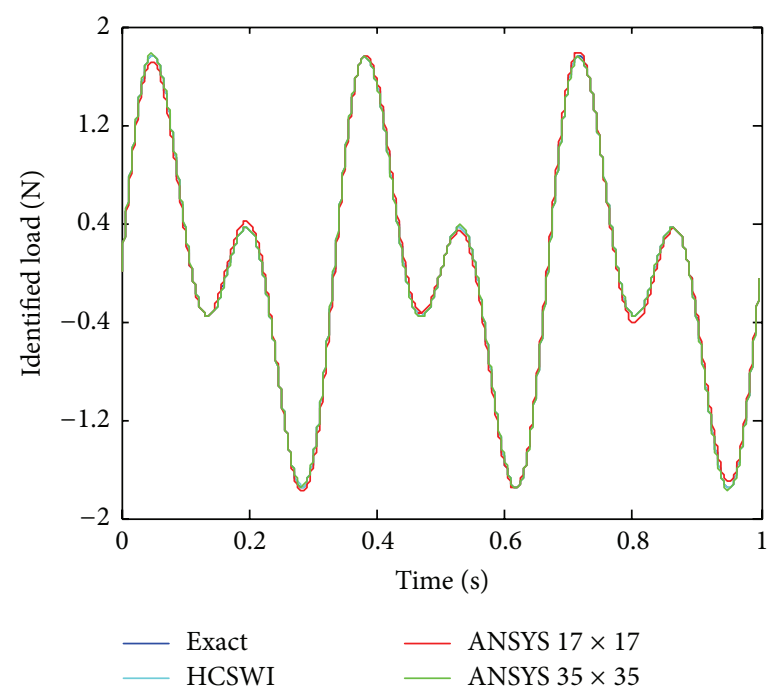

(d1) Identified load $f_{1}$ at node 19

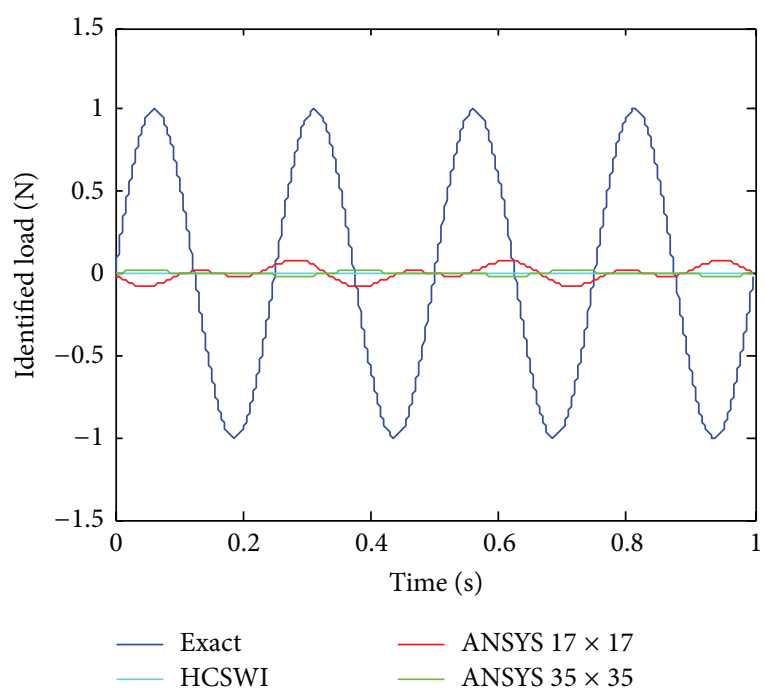

(d2) Identified load $f_{2}$ at node 19

Figure 13: Load identification at different nodes with excitation $f_{1}$ and excitation $f_{2}$ applied at nodes 1 and 9 .

where the submatrix of $\Gamma^{1,1}$ can be calculated by

$\mathbf{A}_{x, y}=\int_{0}^{1} \frac{d x^{\mathrm{T}}}{d \xi} \frac{d y}{d \xi} d \xi, \quad x, y=\varphi_{1}, \psi_{1}, \ldots, \psi_{j-1}$.

Given (6), the nondiagonal submatrix in (28) is zero:

$$
\Gamma^{1,1}=\left[\begin{array}{cccc}
\mathbf{A}_{\varphi_{1}, \varphi_{1}} & 0 & \cdots & 0 \\
0 & \mathbf{A}_{\psi_{1}, \psi_{1}} & \cdots & 0 \\
0 & 0 & \ddots & \vdots \\
0 & 0 & 0 & \mathbf{A}_{\psi_{j-1}, \psi_{j-1}}
\end{array}\right] .
$$

Equation (30) reveals that only the submatrices $\mathbf{A}_{x, y},\left(x=y=\psi_{1}, \ldots, \psi_{j-1}\right)$ will be calculated while other submatrices remain zero when scale 1 is lifted to $j-1$. This can enormously improve the calculating efficiency.

$\Gamma_{2}^{i, j}(i, j=1,2)$ is similar to $\Gamma_{1}^{i, j}(i, j=1,2)$, when $l_{e x}$ and $d \xi$ are replaced by $l_{e y}$ and $d \eta$, respectively.

Based on the orthogonal characteristic of the wavelet bases with respect to the given inner product, the corresponding multiscale equation will be decoupled across total or partial levels and the wavelet bases suit the nesting approximation.

\section{Load Identification}

One can make use of Mindlin plate stiffness matrix and mass matrix and Laplace transform technique to obtain the FRF and then calculate the load.

Equation of motion can be easily discretized and described as follows:

$$
\mathbf{M} \ddot{x}+\mathbf{C} \dot{x}+\mathbf{K} x=\mathbf{F},
$$

where $\mathbf{C}$ is the global damping matrix, $\mathbf{F}$ is a global excitation load vector, which is associated with time, and $\ddot{x}, \dot{x}$, and $x$ stand for the acceleration, velocity, and displacement vectors, respectively. Usually, the damping matrix $\mathbf{C}$ can be expressed as a Rayleigh damping if necessary.

By using the Laplace transform technique, one can obtain

$$
\left(\mathbf{M} s^{2}+\mathbf{C} s+\mathbf{K}\right) \bar{\delta}(s)=\overline{\mathbf{F}}(s),
$$

where

$$
\bar{\delta}(s)=\mathbf{H}_{d}(s) \overline{\mathbf{F}}(s),
$$

where $\mathbf{H}_{d}(s)$ is the FRF matrix of the structure

$$
\mathbf{H}_{d}(s)=\left(\mathbf{M} s^{2}+\mathbf{C} s+\mathbf{K}\right)^{-1} .
$$

Through the modal shape and natural frequency of the structure, the FRF can be obtained. Due to the orthogonality in modal shape, we can obtain

$$
\begin{array}{r}
\mathbf{I}=\widetilde{\boldsymbol{\Phi}}^{\mathrm{T}} \mathbf{M} \widetilde{\boldsymbol{\Phi}} \\
\operatorname{diag}\left(2 \xi_{i} \omega_{i}\right)=\widetilde{\boldsymbol{\Phi}}^{\mathrm{T}} \mathbf{C} \widetilde{\boldsymbol{\Phi}} \\
\operatorname{diag}\left(\omega_{i}^{2}\right)=\widetilde{\boldsymbol{\Phi}}^{\mathrm{T}} \mathbf{K} \widetilde{\boldsymbol{\Phi}},
\end{array}
$$

where diag is the diagonal matrix, $\widetilde{\boldsymbol{\Phi}}$ is the modal shape, $\xi_{i}$ is the damping ratio, and $\omega_{i}$ is the natural frequency.

According to (35), we can obtain

$$
\begin{aligned}
& \mathbf{M}=\left(\widetilde{\boldsymbol{\Phi}}^{\mathrm{T}}\right)^{-1} \widetilde{\boldsymbol{\Phi}}^{-1} \\
& \mathbf{C}=\left(\widetilde{\boldsymbol{\Phi}}^{\mathrm{T}}\right)^{-1} \operatorname{diag}\left(2 \xi_{i} \omega_{i}\right) \widetilde{\boldsymbol{\Phi}}^{-1} \\
& \mathbf{K}=\left(\widetilde{\boldsymbol{\Phi}}^{\mathrm{T}}\right)^{-1} \operatorname{diag}\left(\omega_{i}^{2}\right) \widetilde{\boldsymbol{\Phi}}^{-1}
\end{aligned}
$$






$\begin{array}{ll}\text { HCSWI } & \text { ANSYS } 35 \times 35 \\ \text { ANSYS } 17 \times 17 & - \text { A }\end{array}$

(a) Identified load $f_{1}$ at node 2

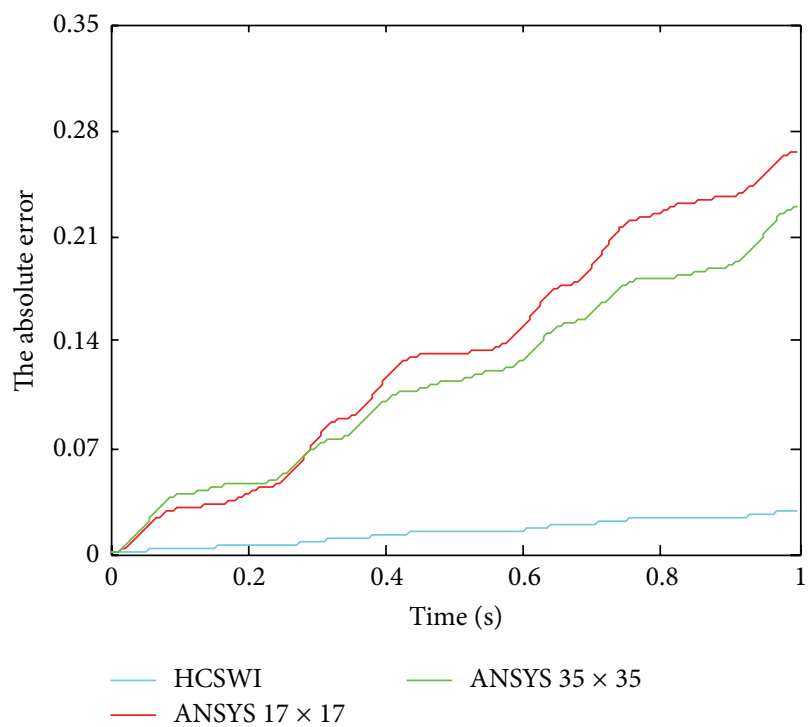

(c) Identified load $f_{2}$ at node 18

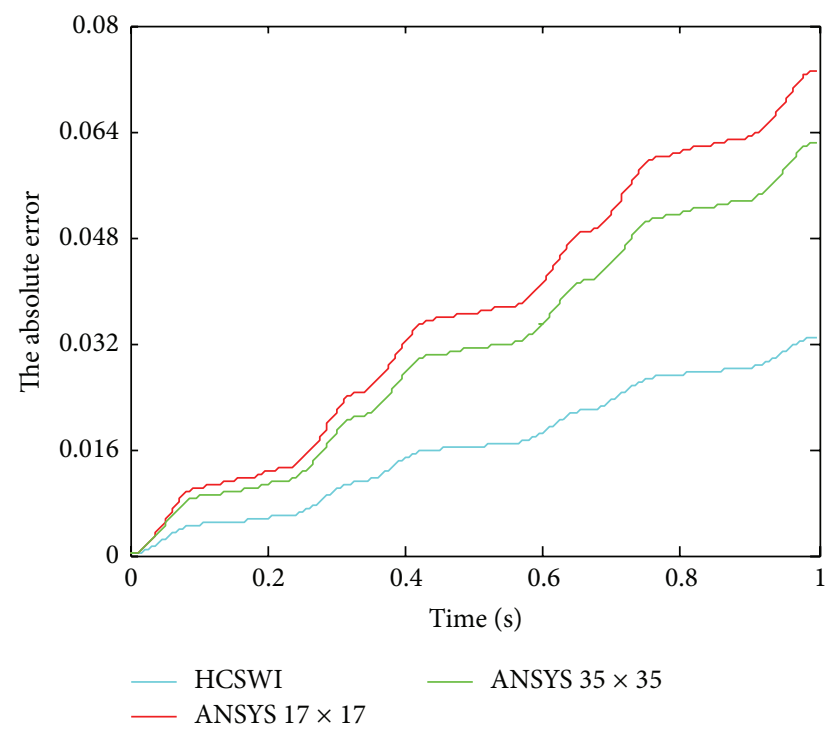

(b) Identified load $f_{2}$ at node 10

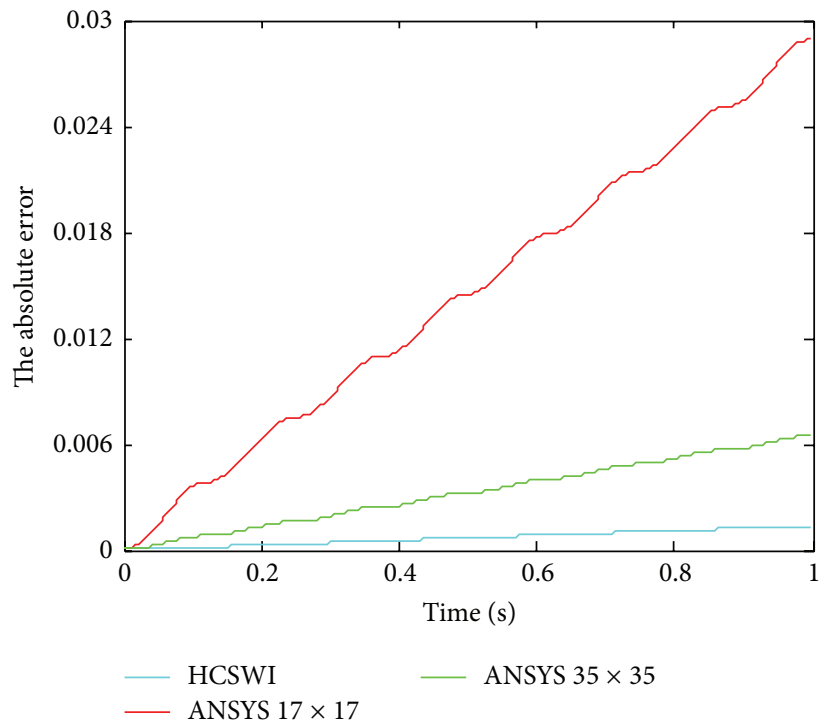

(d) Identified load $f_{1}$ at node 19

FIgURE 14: The absolute error with excitation $f_{1}$ and excitation $f_{2}$ applied at nodes 1 and 9 .

Substituting (36) into (34), we can obtain

$$
\begin{aligned}
\mathbf{H}_{d}(s) & =\left[\left(\widetilde{\boldsymbol{\Phi}}^{\mathrm{T}}\right)^{-1} \operatorname{diag}\left(s^{2}+2 \xi_{i} \omega_{i} s+\omega_{i}^{2}\right) \widetilde{\boldsymbol{\Phi}}^{-1}\right]^{-1} \\
& =\widetilde{\boldsymbol{\Phi}} \operatorname{diag}\left(\frac{1}{\left(s^{2}+2 \xi_{i} \omega_{i} s+\omega_{i}^{2}\right)}\right) \widetilde{\boldsymbol{\Phi}}^{\mathrm{T}} \\
& =\sum_{i=1}^{n} \frac{\widetilde{\phi}_{i} \widetilde{\phi}_{i}^{\mathrm{T}}}{s^{2}+2 \xi_{i} \omega_{i} s+\omega_{i}^{2}} .
\end{aligned}
$$

Assuming $s=j \omega(j=\sqrt{-1})$, then the FRF matrix can be obtained:

$$
\mathbf{H}_{d}(\omega)=\left.\mathbf{H}_{d}(\mathrm{~s})\right|_{s=j \omega}=\sum_{i=1}^{n} \frac{\widetilde{\phi}_{i} \widetilde{\phi}_{i}^{\mathrm{T}}}{\omega_{i}^{2}-\omega^{2}+j 2 \xi_{i} \omega_{i} \omega} .
$$

Every element $\mathbf{H}_{d}^{m n}(\omega)$ represents the $n$ point excitation and the $m$ point response of the FRF [7]. The expression is

$$
\mathbf{H}_{d}^{m n}(\omega)=\sum_{i=1}^{n} \frac{\widetilde{\phi}_{i}^{m} \widetilde{\phi}_{i}^{n}}{\omega_{i}^{2}-\omega^{2}+j 2 \xi_{i} \omega_{i} \omega} .
$$

And load identification equation can be written as

$$
\mathbf{F}=\mathbf{H}_{d}{ }^{-1} \mathbf{x} \text {. }
$$

In this way, the load identification problem of Mindlin plate can be solved. The main idea of load identification is to use the FRF and the measured structural response signals to determine the excitation load. However, the inverse matrix of the FRF is ill-posed. However, select singular values are the key factors in solving the inverse problem. In this paper, the Lcurve of regularization methods is used to choose the singular 


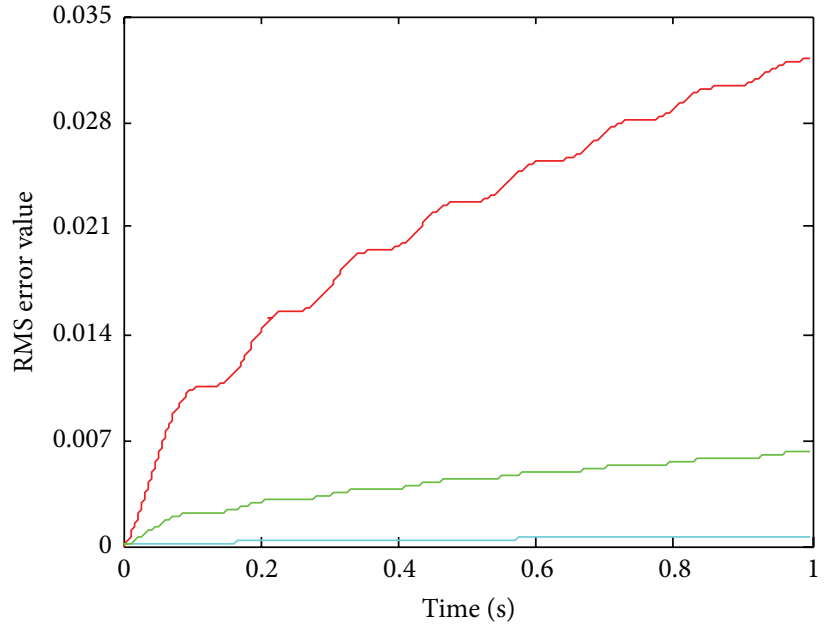

- HCSWI

ANSYS $17 \times 17$

(a) Identified load $f_{1}$ at node 2



HCSWI

- ANSYS $35 \times 35$

ANSYS $17 \times 17$

(c) Identified load $f_{2}$ at node 18

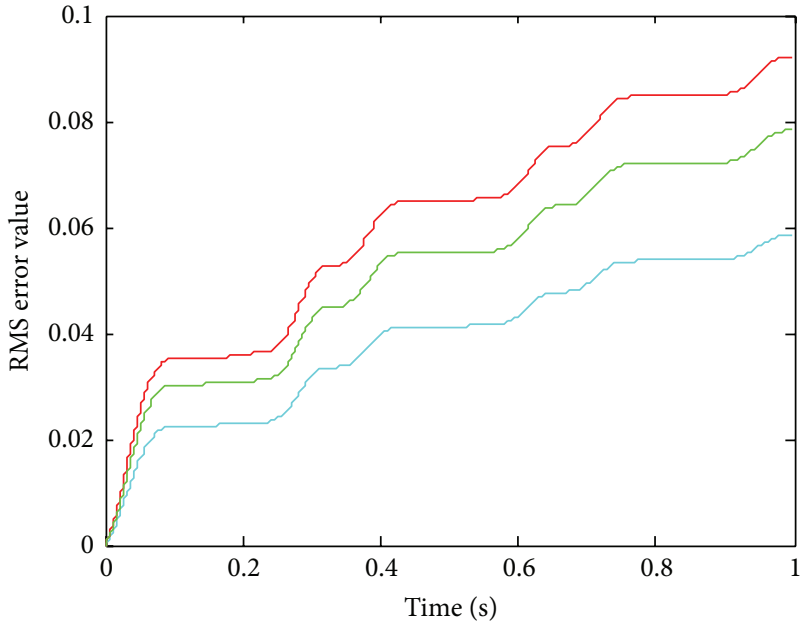

- HCSWI

— ANSYS $35 \times 35$

ANSYS $17 \times 17$

(b) Identified load $f_{2}$ at node 10



HCSWI $\quad$ ANSYS $35 \times 35$

ANSYS $17 \times 17$

(d) Identified load $f_{1}$ at node 19

Figure 15: The root mean square error value with excitation $f_{1}$ and excitation $f_{2}$ applied at nodes 1 and 9 .
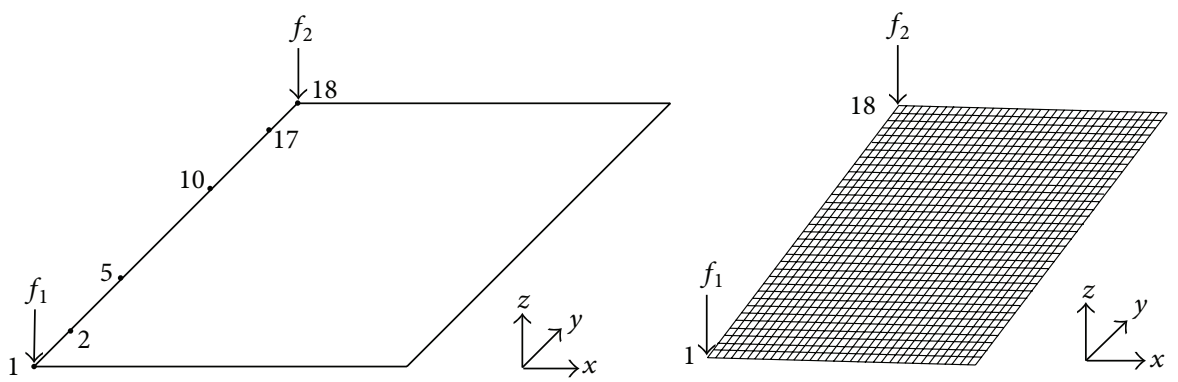

FIGURE 16: The location of $f_{1}$ and $f_{2}$ in the Mindlin plate. 

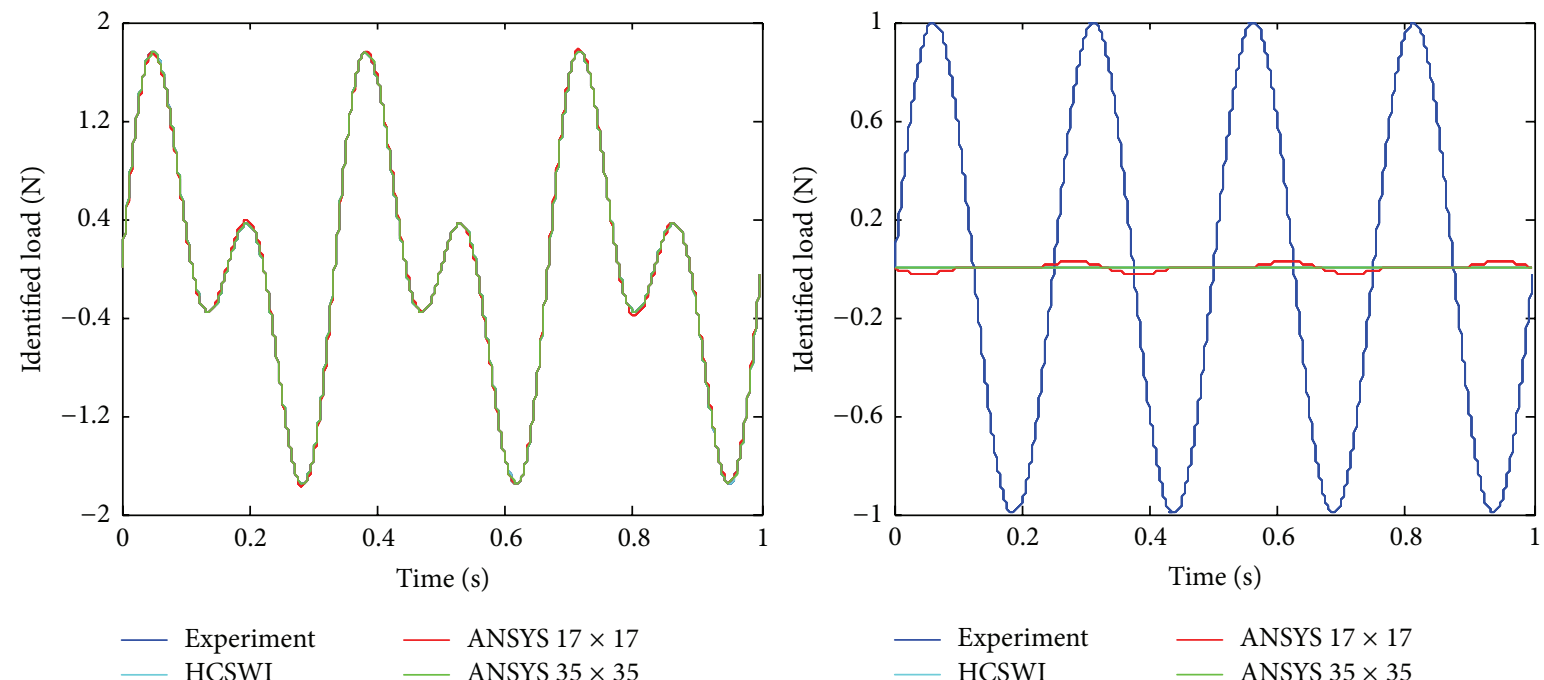

(a1) Identified load $f_{1}$ at node 2

(a2) Identified load $f_{2}$ at node 2
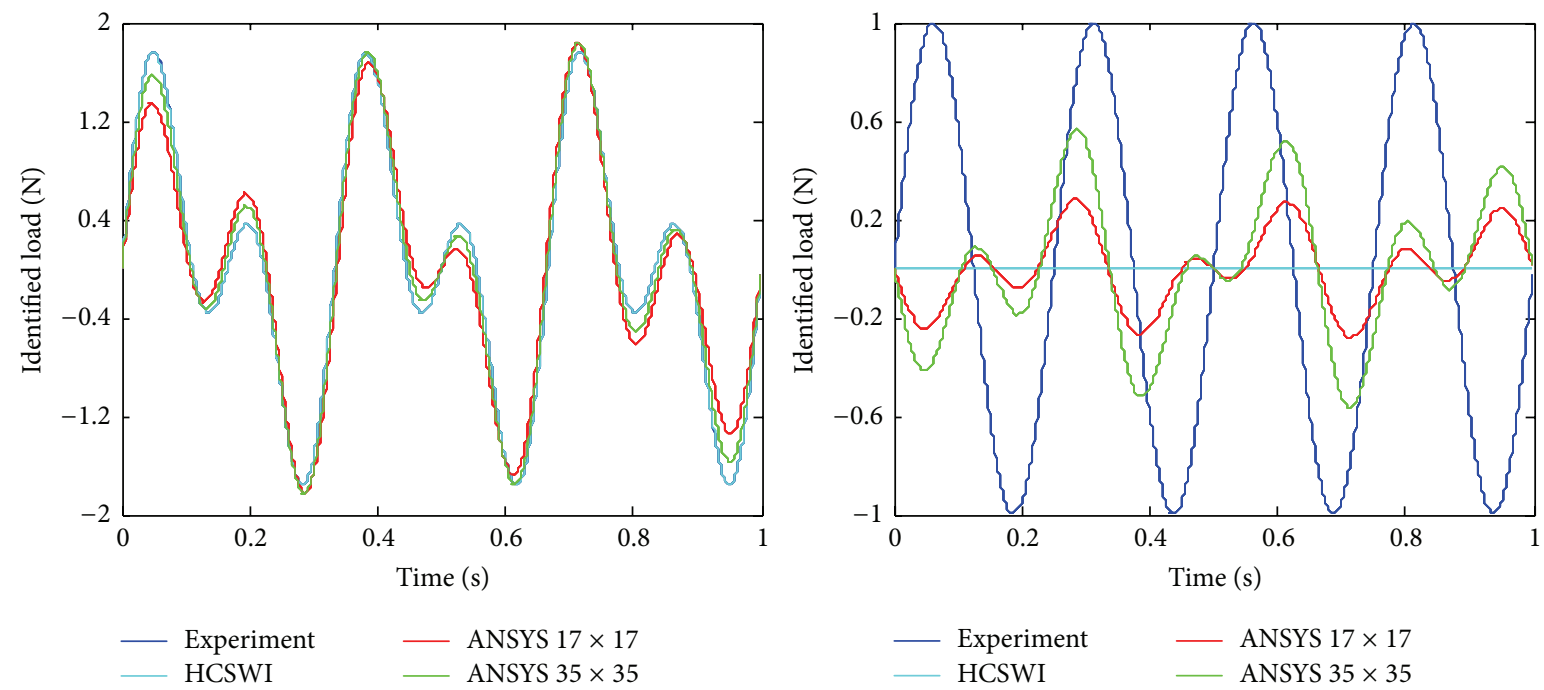

(b1) Identified load $f_{1}$ at node 5

(b2) Identified load $f_{2}$ at node 5

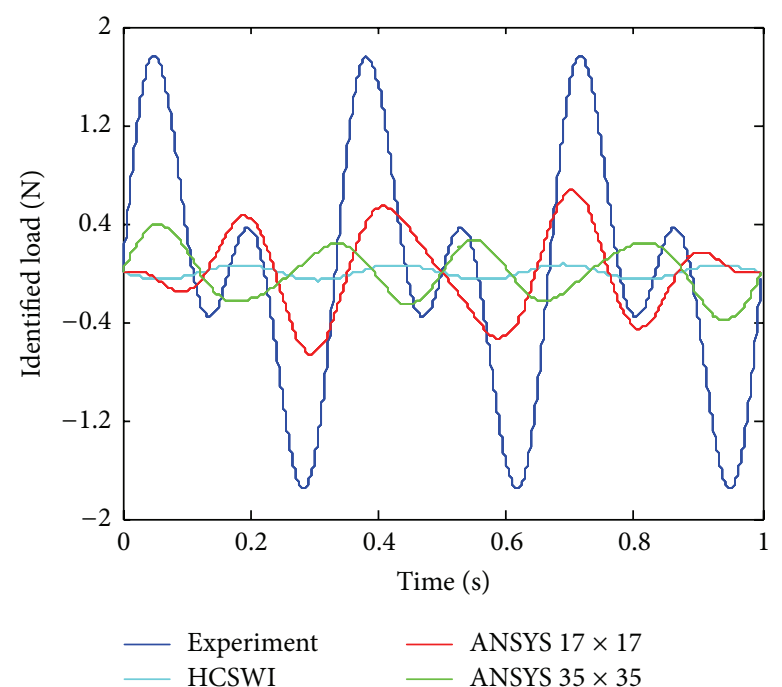

(c1) Identified load $f_{1}$ at node 10

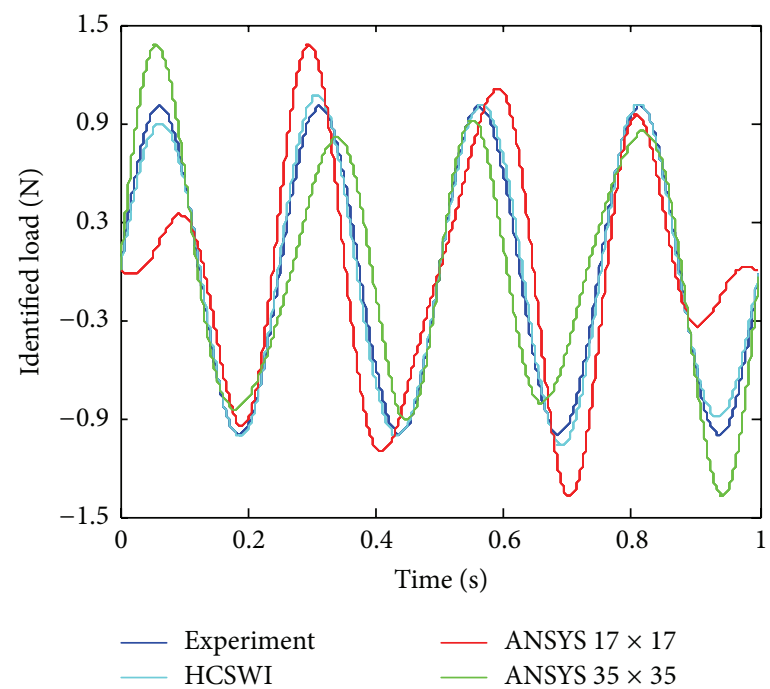

(c2) Identified load $f_{2}$ at node 10

FIGURE 17: Continued. 


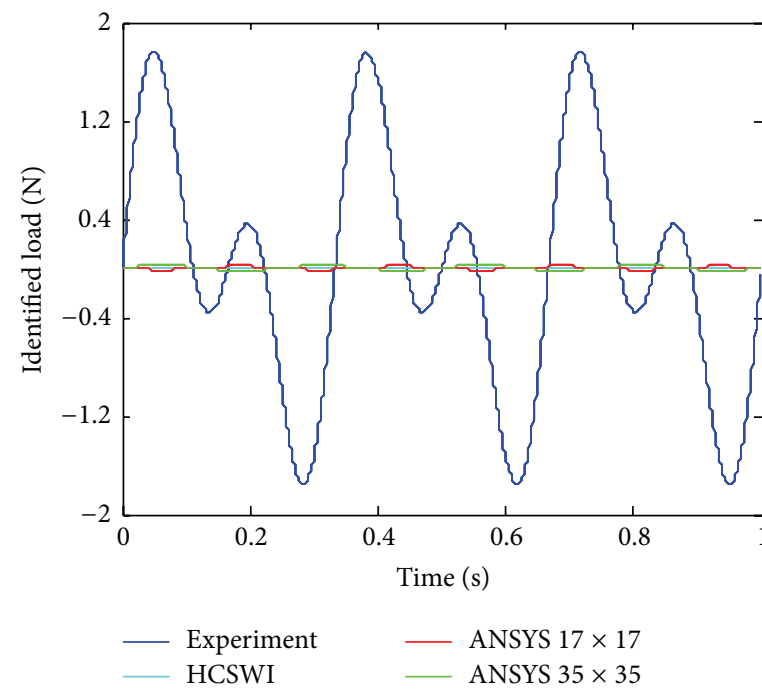

(d1) Identified load $f_{1}$ at node 17

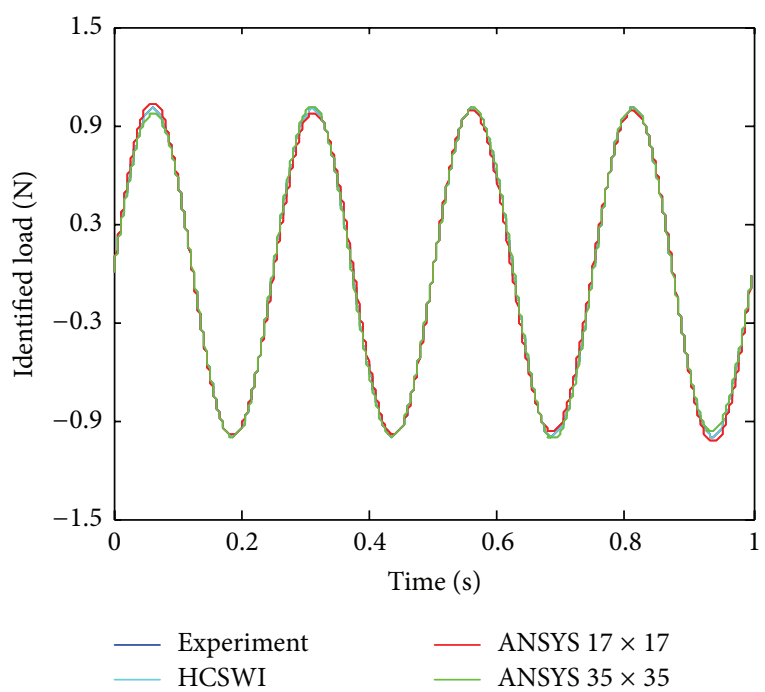

(d2) Identified load $f_{2}$ at node 17

FIgURE 17: Load identification at different nodes with excitation $f_{1}$ and excitation $f_{2}$ applied at nodes 1 and 18 .

values [33]. Therefore, the method of SVD is adopted to solve the problem.

SVD is a useful tool that will help one to prove the nature of the deconvolution problem. The SVD of $\mathbf{H}_{d}$ is defined as follows:

$$
\mathbf{H}_{d}=\mathbf{U} \Sigma \mathbf{V}^{\mathrm{T}}=\sum_{i=1}^{n} u_{i} \sigma_{i} v_{i}^{\mathrm{T}}
$$

where $\mathbf{U}$ is the matrix of left singular vectors, $\mathbf{V}$ is the matrix of right singular vectors, and diagonal elements of matrix $\Sigma$ are the singular values of $\mathbf{H}_{d}$.

The solution of (40) can be rewritten in a term of SVD:

$$
\mathbf{F}=\sum_{i=1}^{n} \frac{u_{i}^{\mathrm{T}} \mathbf{x}}{\sigma_{i}} v_{i}
$$

The singular values will help one to determine the nature of the problems. The FRF and the measured structural response signals can solve the excitation load.

The equation of absolute error of the identified load is calculated as follows:

$$
e_{\mathrm{ma}}=\frac{1}{n} \sum_{i=1}^{n}\left|\mathbf{F}_{\mathrm{id}}-\mathbf{F}_{\text {real }}\right| .
$$

The root mean square (RMS) error value of the identified load can be given by

$$
e_{\mathrm{rms}}=\sqrt{\frac{\sum_{i=1}^{n}\left(\mathbf{F}_{\text {id }}-\mathbf{F}_{\text {real }}\right)^{2}}{n}} .
$$

\section{Numerical Examples}

It is well known that the vibration structures are complex in engineering. In this section, we involve different excitations cases to simulate the load identification in the Mindlin plate. The Mindlin plate dimension is as follows: length $1 \mathrm{~m}$, width $1 \mathrm{~m}$, and thickness $0.01 \mathrm{~m}$. The material is assumed as aluminum, with Young's modulus $70 \mathrm{GPa}$, Poisson ratio 0.3, and density $2730 \mathrm{~kg} / \mathrm{m}^{3}$. We fixed the right of the Mindlin plate. A computer is used with an Intel CPU of $3.1 \mathrm{GHz}$ and a memory of $4 \mathrm{G}$ bytes, 64 -bit Windows 7 operating system, and Matlab. The responses of different nodes are substituted into (40) to reconstruct the load. Load identification is implemented under an undamped condition. The excitations are used as exact solutions.

Commercial software ANSYS 14.5 is used for modeling, and the shell 43 elements are selected. The mesh of the Mindlin plate is shown in Figure 6. The geometric modeling and material parameters of the Mindlin plate are input into the software ANSYS. Harwell-Boeing method is utilized to extract the stiffness and mass matrix. Three DOFs at each node are retained, that is, $\theta_{i x}, \theta_{i y}, \omega_{i}\left(i=1,2, \ldots,(n+1)^{2}\right)$. Substituting the stiffness and mass matrix into (40), we can obtain the load identification results of ANSYS. The load identification results of grids $17 \times 17$ and $35 \times 35$ are compared with that of HCSWI element.

4.1. Load Identification with Single Excitation. Figure 7 shows that the location of the excitation has been applied at node 1. The sampling frequency studied is $1000 \mathrm{~Hz}$ and the time duration of measurement is $1 \mathrm{~s}$. The signal-to-noise ratio (SNR) of the excitation signal is $130 \mathrm{~dB}$. The applied load is used to obtain the displacement of nodes. The displacements of nodes 2, 9, 18, 19, 20, and 145 can be substituted into a HCSWI element and $17 \times 17,35 \times 35$ ANSYS elements for load reconstruction. Comparison between the precision of HCSWI element and those of ANSYS element verifies the superiority of the former. The excitation $f=\sin (6 \times$ $\pi t)$ is assumed to act in the Mindlin plate as Figure 7 shows. 




HCSWI - ANSYS $17 \times 17$

(a) Identified load $f_{1}$ at node 2

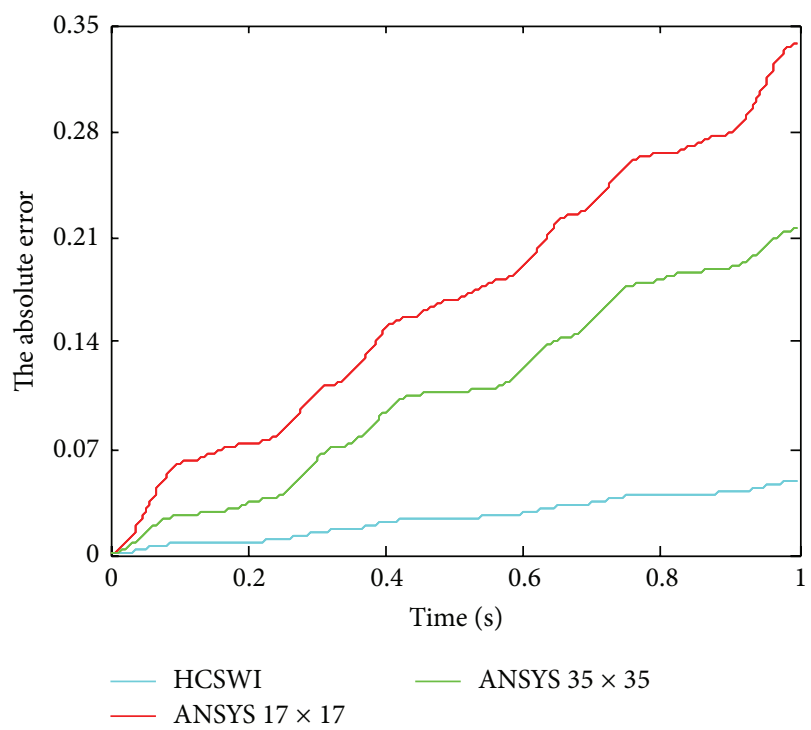

(c) Identified load $f_{2}$ at node 18

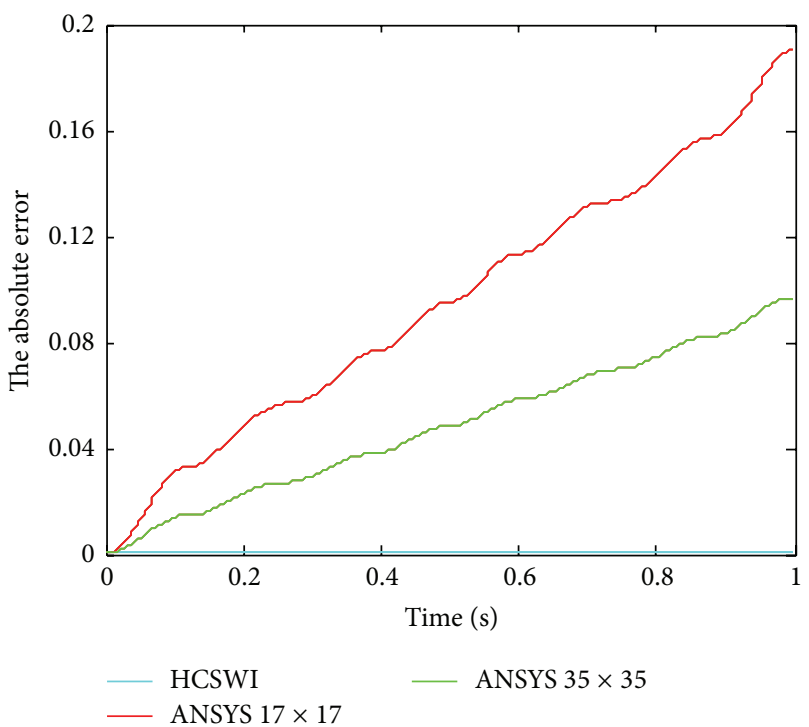

(b) Identified load $f_{2}$ at node 10

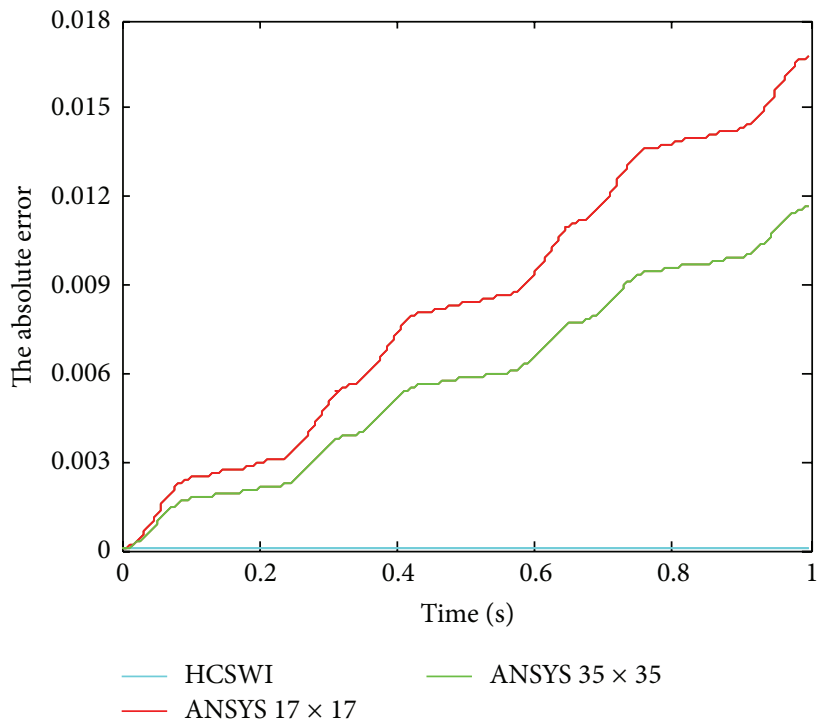

(d) Identified load $f_{1}$ at node 19

Figure 18: The absolute error with excitation $f_{1}$ and excitation $f_{2}$ applied at nodes 1 and 18 .

Figure 8 shows the load identification results at nodes 2 , $9,18,19,20$, and 145 . The high precision is obtained at nodes 2,19 , and 20 . When the response nodes such as 9,145 , and 18 are far away from node 1 where the load is applied, the error is great. It can be seen that when the response node is close to node 1 , the load identification has a high precision. Obviously, HCSWI element has the highest precision.

Figures 9 and 10 show the absolute error and the RMS error value by using HCSWI and ANSYS $17 \times 17$ and $35 \times$ 35. It can be seen that HCSWI element error line is almost horizontal. And ANSYS $35 \times 35$ error is lower than that of 17 $\times 17$. The accuracy of ANSYS is gradually improved with the increasing number of grids. The result verifies the superiority of HCSWI element.

Figure 11 shows the FRF between the different response nodes and applied node. In this paper we construct the high precision FRF by using HCSWI element. The FRF has a high precision and can calculate the load in engineering practice.

4.2. Load Identification with Two Excitations. Figure 12 shows the location of the excitation signals applied at nodes 1 and 9. Compared with ANSYS results, HCSWI element can accurately identify the applied load, which verifies its superiority. The excitations $f_{1}=\sin (6 \times \pi t)+\sin (12 \times \pi t)$ and $f_{2}=\sin (8 \times \pi t)$ are assumed to act in the Mindlin plate as shown in Figure 12.

Figure 13 shows the load identification at nodes 2, 10, 18 , and 19. Because node 2 or 19 is close to the location of applied load $f_{1}$ and away from that of $f_{2}$, the response node 2 or 19 can accurately identify $f_{1}$ but cannot identify $f_{2}$. For the same reason, the response at node 10 can accurately identify $f_{2}$ but cannot identify $f_{1}$. Figures 14 and 15 show 




(a) Identified load $f_{1}$ at node 2

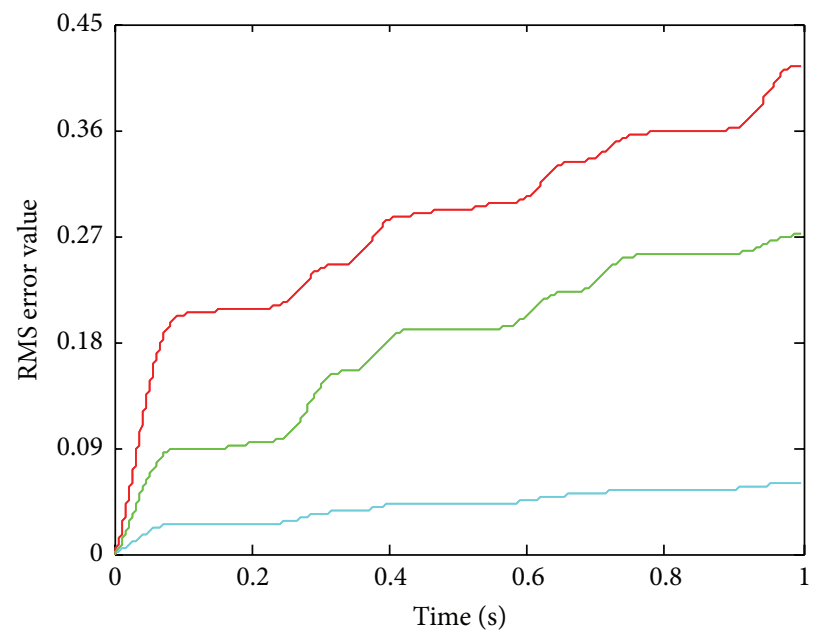

HCSWI

ANSYS $17 \times 17$

(c) Identified load $f_{2}$ at node 18



(b) Identified load $f_{2}$ at node 10



HCSWI $\quad$ ANSYS $35 \times 35$

- ANSYS $17 \times 17$

(d) Identified load $f_{1}$ at node 19

FIgURE 19: The root mean square error value with excitation $f_{1}$ and excitation $f_{2}$ applied at nodes 1 and 18 .

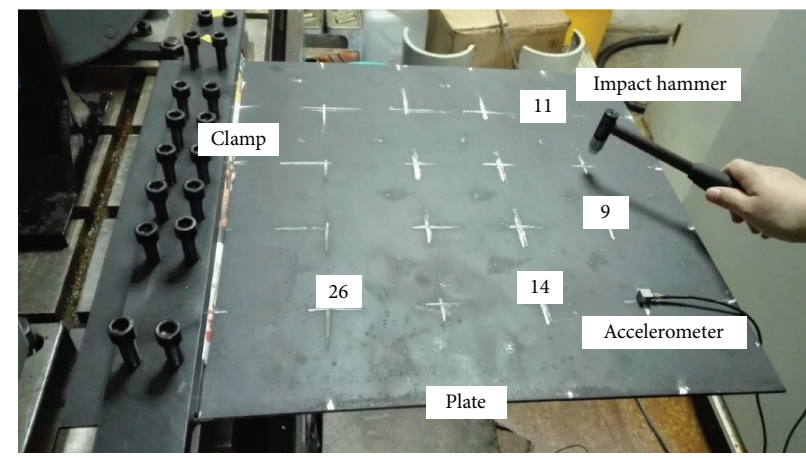

FIgURE 20: The Mindlin plate structure applied for load identification. 


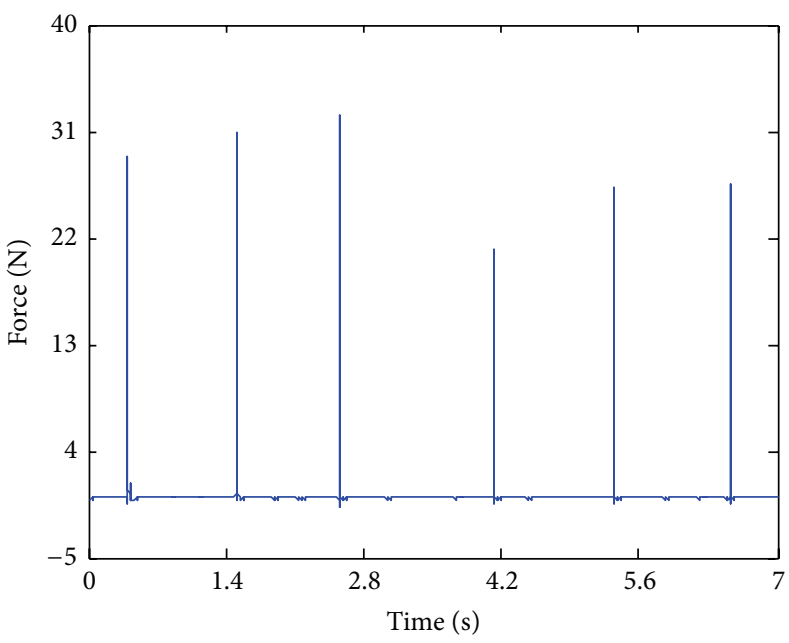

(a)

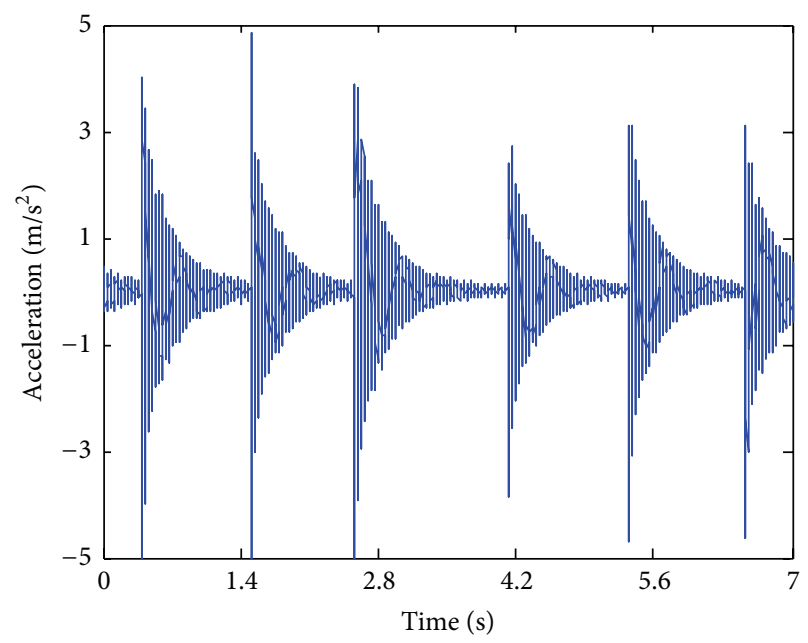

(b)

FIGURE 21: The measurement data from force transducer and accelerometer: (a) impact loads and (b) acceleration responses.

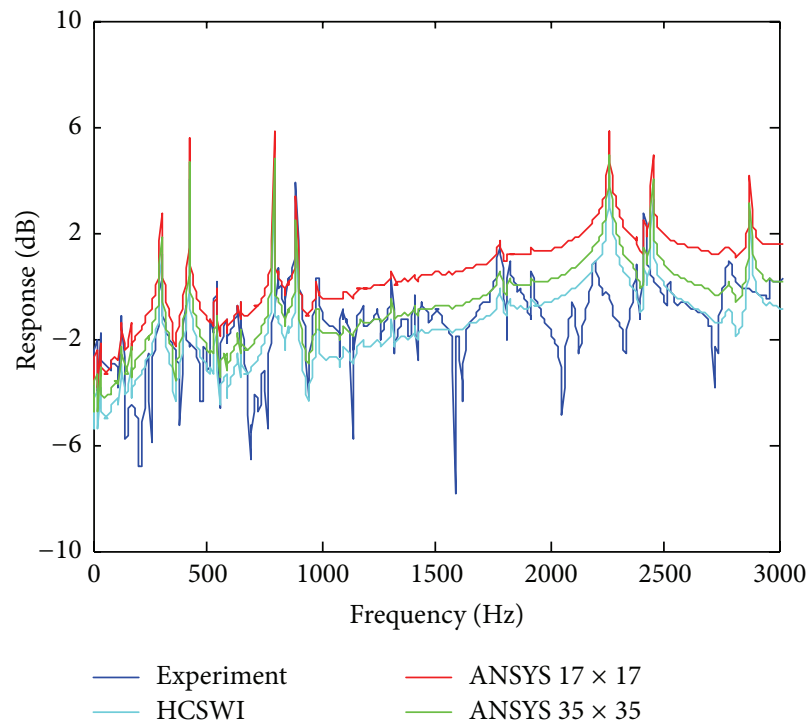

(a) Node 9



(b) Node 26

FIGURE 22: Acceleration FRF with the excitation at nodes 9 and 26 and the response at node 8 .

that HCSWI element has higher accuracy than ANSYS elements.

Figure 16 shows the location of the excitation signals applied at nodes 1 and 18. Figure 17 shows the load identification at nodes $2,5,10$, and 17 . Because node 2 is close to the location of applied load $f_{1}$ and away from that of $f_{2}$, the response at node 2 can accurately identify $f_{1}$ but cannot identify $f_{2}$. For the same reason, the response node 17 can accurately identify $f_{2}$ but cannot identify $f_{1}$. Figures 18 and 19 show that the accuracy of HCSWI element is higher than ANSYS elements.

\section{Experimental Verification}

The Mindlin plate structure for load identification is set up as shown in Figure 20. The plate has been used by
Qiao et al. for impact and harmonic force identification [34]. The Mindlin plate dimension is as follows: length $0.5 \mathrm{~m}$, width $0.5 \mathrm{~m}$, and height $0.005 \mathrm{~m}$. The material is number 45 steel with Young's modulus $206 \mathrm{GPa}$, Poisson ratio 0.3 , and density $7917 \mathrm{~kg} / \mathrm{m}^{3}$. The left of the Mindlin plate is fixed. In the impact experiment, the excitation which is marked as $F$ in Figure 21(a) is continuously impacted. The Mindlin plate is divided into $5 \times 5$ elements $(6 \times$ 6 nodes). The excitation locations are at nodes 9 and 26 . The accelerometer sensor is mounted at node 8 . For the purposes of load identification validations, the force signals are recorded by the force transducers inserted in the impact hammer. The response signals are recorded by accelerometer sensor at the selected node 8 . Therefore, the forces acting on the location at nodes 9 and 26 can be constructed from the response of the selected node 8 . With the acceleration 




(a) Identified load $f$ at node 9

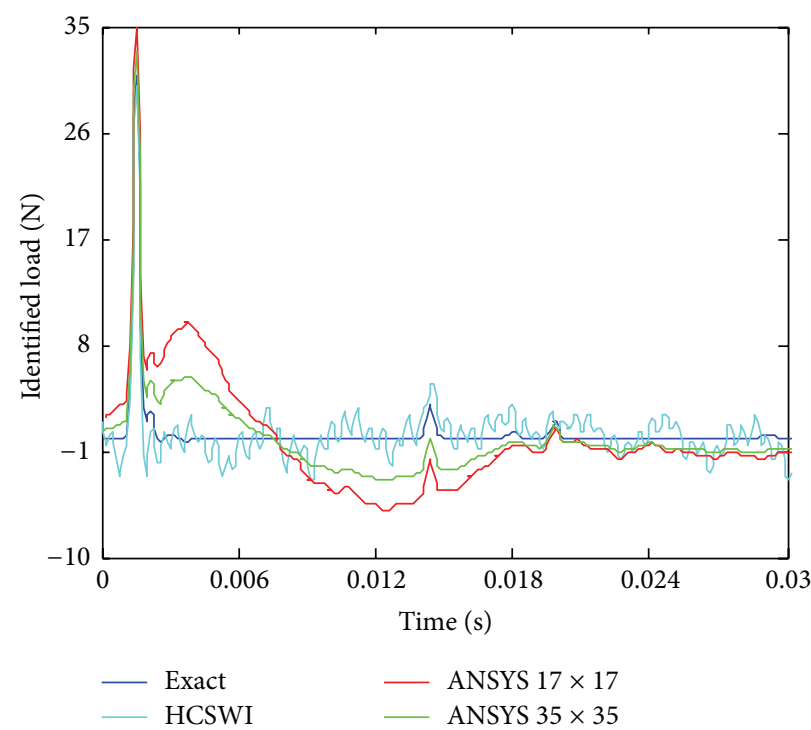

(b) Identified load $f$ at node 26

Figure 23: Load identification at node 8 with excitation $f$ applied at nodes 9 and 26.

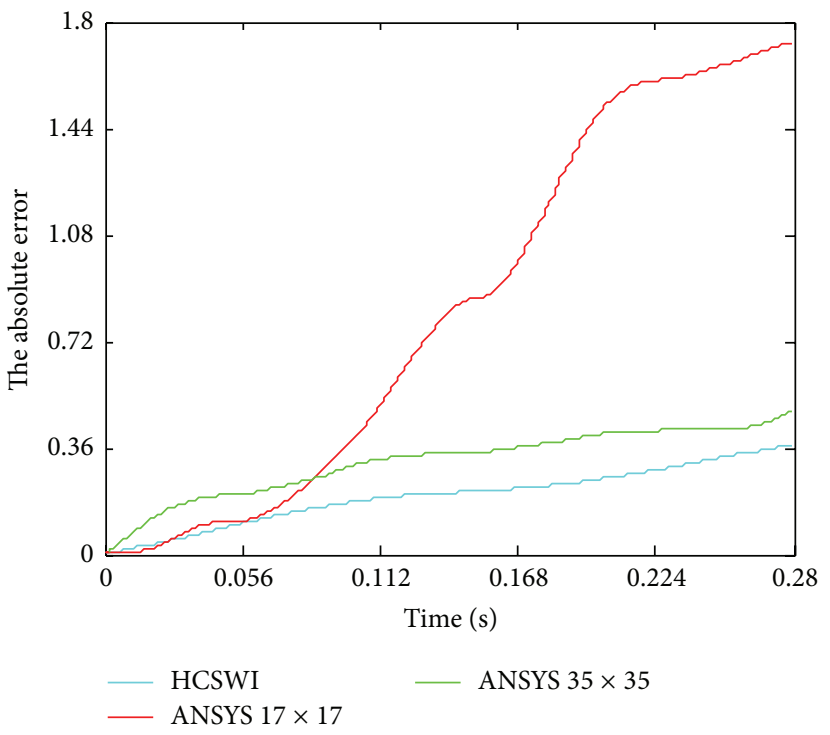

(a) Identified load $f$ at node 9

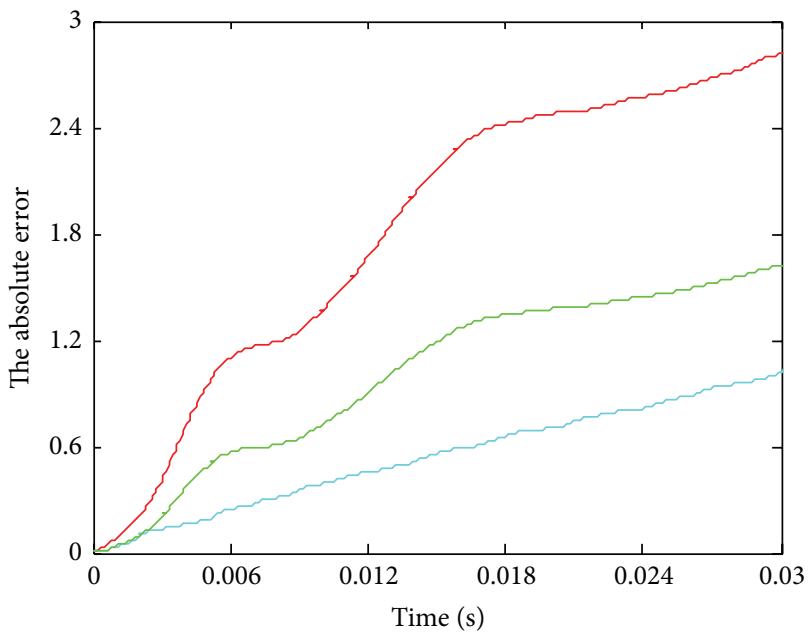

HCSWI

- ANSYS $35 \times 35$

- ANSYS $17 \times 17$

(b) Identified load $f$ at node 26

FIgURE 24: The absolute error with excitation $f$ applied at nodes 9 and 26.

response, the forces can be identified by HCSWI and ANSYS elements.

A modal impact hammer ( $\mathrm{PCB} 086 \mathrm{C} 01$ with sensitivity $12.37 \mathrm{mV} / \mathrm{N}$ ) is used to impose impact forces. In the impact experiment, the Mindlin plate is impacted many times. Accelerometers sensor and force channels are synchronously sampled by LMSSCADASIII data acquisition system with a sampling frequency of $4096 \mathrm{~Hz}$ and spectral line of 1025 . At each excitation, the FRF is measured. After the inverse fast Fourier transform, the data length of the discrete impulse response function is 2050. In this paper, we use SVD to solve the ill-posed problem since it is an efficient algorithm.
The structure is assumed to exhibit Rayleigh damping as follows:

$$
\mathbf{C}=a_{1} \mathbf{M}+a_{2} \mathbf{K},
$$

where $a_{1}$ and $a_{2}$ are the damping coefficients.

During the impact experiment, impact excitations are continuously recorded. Each case is impacted using a modal impact hammer to apply and measure the load, while the acceleration response is measured using an accelerometer sensor described previously. Figure 21 shows the original impact loads acting on the location of node 9 and the acceleration response at node 8 . It can be seen from Figure 21 that impact loads have a typical impulsive profile and the 




(a) Identified load $f$ at node 9

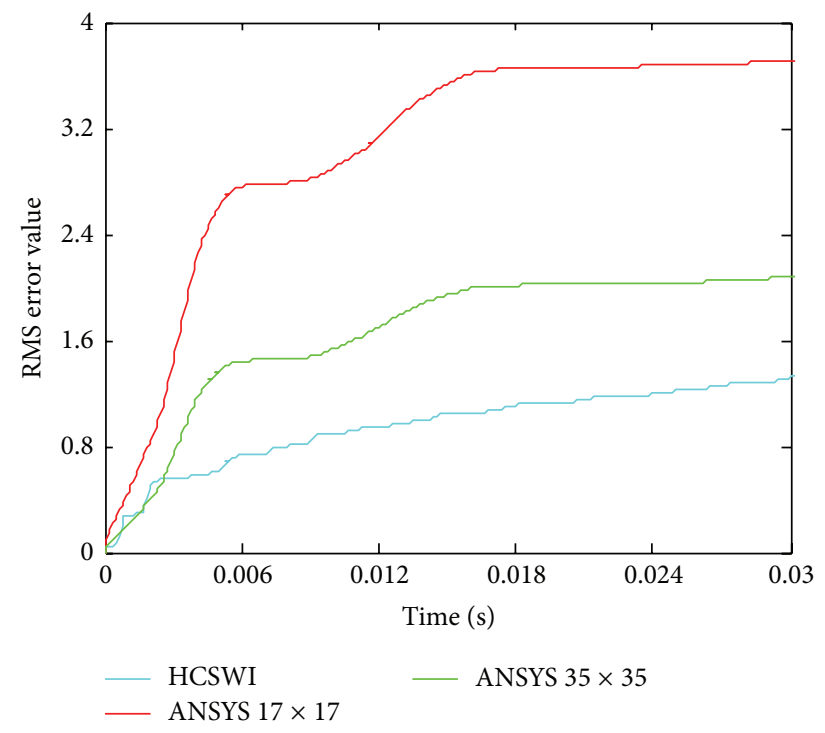

(b) Identified load $f$ at node 26

Figure 25: The root mean square error value with excitation $f$ applied at nodes 9 and 26.

response of each impact case displays a rapid attenuation tendency.

One can correct the elastic moduli; those are deduced from the characteristic value of the Mindlin plate [35-38]. The corresponding elastic modulus value $E_{i}^{m}(i=1,2, \ldots)$ is solved for each frequency, which makes the experiments frequency values equal to calculating frequency values of FEM. The equation of the corrected elastic modulus can be given by

$$
\left|\omega_{i}^{2} \mathbf{M}-E_{i}^{m} \frac{\mathbf{K}}{E}\right|=0
$$

The experiment measures the acceleration frequency response functions. The following equation is the acceleration frequency response functions solved by FEM. The accuracy of HCSWI can be verified by comparing the FRF of HCSWI and ANSYS elements with the experiment data:

$$
\mathbf{H}_{a}^{m n}(w)=\sum_{i=1}^{n} \frac{-\omega^{2} \widetilde{\phi}_{i}^{m} \widetilde{\phi}_{i}^{n}}{\omega_{i}^{2}-\omega^{2}+j 2 \xi_{i} \omega_{i} \omega} .
$$

Figure 22 shows the acceleration FRF at nodes 8, 9, 8, and 26 after the model correction. As shown in Figure 22, the result of HCSWI is the closest to the experimental acceleration FRF. With the increase of ANSYS grid, the frequency responses of ANSYS are close to the experimental results.

Figure 23 shows the load identification results at nodes 9 and 26. When the excitation node such as 26 is far away from node 8 where the accelerometer sensor is mounted, the error is great. It can be seen that when the excitation node is close to node 8 , the load identification has a high precision. Obviously, HCSWI element has the highest precision.

Figures 24 and 25 show the absolute error and the RMS error value by using HCSWI and ANSYS $17 \times 17$ and $35 \times$ 35. It can be seen that HCSWI element error line is lower than result of ANSYS. And ANSYS $35 \times 35$ error is lower than that of $17 \times 17$. The accuracy of ANSYS is gradually improved with the increasing number of grids. The result verifies the superiority of HCSWI element.

\section{Conclusion}

The present study constructs a Hermitian Mindlin plate wavelet element. It is substituted into finite element functions to solve the FRF and then derive the loads. Numerical calculations are carried out with different excitation cases in the Mindlin plate. The SVD is adopted to solve ill-posed inverse problem of the load identification. The results of ANSYS shell 43 elements with grids $17 \times 17$ and $35 \times 35$ are compared with that of HCSWI Mindlin plate element. It shows that the accuracy of ANSYS gradually improves with the increasing number of grids. Compared with ANSYS, HCSWI Mindlin plate element can accurately identify the applied load. The load identification has desirable results when the response node is close to the node where the load is applied. The accuracy of HCSWI can be verified by comparing the FRF of HCSWI and ANSYS elements with the experiment data. The FRFs and response spectrums can calculate the loads. The experiment proves that the load identification of HCSWI Mindlin plate is effective and precise. But the frequency-domain requires a long stationary time to calculate the inversion of the FRF matrix at each frequency. The load identification based on FRF is restricted as the result is inaccurate when the frequency content is close to certain resonance frequency of the structure. Hermitian wavelet finite element with high precision can alleviate this problem but cannot fundamentally solve the problem.

\section{Competing Interests}

The authors declare that they have no competing interests. 


\section{Acknowledgments}

This work is supported by National Natural Science Foundation of China (nos. 51421004 and 51405370) and the National Key Basic Research Program of China (no. 2015CB057400). The project is supported by Natural Science Basic Plan in Shaanxi Province of China (no. 2015JQ5184) and Shaanxi Province Postdoctoral Research Project.

\section{References}

[1] L. Wang, X. Han, and Y. Xie, "A new conjugate gradient method for solving multi-source dynamic load identification problem," International Journal of Mechanics and Materials in Design, vol. 9, no. 3, pp. 191-197, 2013.

[2] B. Khalfi and A. Ross, "Influence of partial constrained layer damping on the bending wave propagation in an impacted viscoelastic sandwich," International Journal of Solids and Structures, vol. 50, no. 25-26, pp. 4133-4144, 2013.

[3] I. Senjanović, N. Vladimir, and N. Hadžić, "Modified Mindlin plate theory and shear locking-free finite element formulation," Mechanics Research Communications, vol. 55, pp. 95-104, 2014.

[4] K. Liu, S. S. Law, X. Q. Zhu, and Y. Xia, "Explicit form of an implicit method for inverse force identification," Journal of Sound and Vibration, vol. 333, no. 3, pp. 730-744, 2014.

[5] H. Ronasi, H. Johansson, and F. Larsson, "A numerical framework for load identification and regularization with application to rolling disc problem," Computers \& Structures, vol. 89, no. 1-2, pp. 38-47, 2011.

[6] S. Q. Wu and S. S. Law, "Moving force identification based on stochastic finite element model," Engineering Structures, vol. 32, no. 4, pp. 1016-1027, 2010.

[7] Z. Li, Z. Feng, and F. Chu, "A load identification method based on wavelet multi-resolution analysis," Journal of Sound and Vibration, vol. 333, no. 2, pp. 381-391, 2014.

[8] Z.-B. Yang, M. Radzienski, P. Kudela, and W. Ostachowicz, "Two-dimensional modal curvature estimation via Fourier spectral method for damage detection," Composite Structures, vol. 148, pp. 155-167, 2016.

[9] H. Van der Auweraer, P. Mas, P. Peeters, K. Janssens, and A. Vecchio, "Modal and path contribution models from in-operation data: review and new approaches," Shock and Vibration, vol. 15, no. 3-4, pp. 403-411, 2008.

[10] B. Qiao, X. Chen, X. Luo, and X. Xue, "A novel method for force identification based on the discrete cosine transform," Journal of Vibration and Acoustics, vol. 137, no. 5, Article ID 051012, 15 pages, 2015.

[11] C. Ma and H. Hua, "Force identification technique by the homotopy method," Journal of Mechanical Science and Technology, vol. 29, no. 10, pp. 4083-4091, 2015.

[12] B. Qiao, X. Zhang, X. Luo, and X. Chen, "A force identification method using cubic B-spline scaling functions," Journal of Sound and Vibration, vol. 337, pp. 28-44, 2015.

[13] B. Qiao, X. Chen, X. Xue, X. Luo, and R. Liu, "The application of cubic B-spline collocation method in impact force identification," Mechanical Systems and Signal Processing, vol. 64-65, pp. 413-427, 2015.

[14] Y. Liu and W. S. Shepard Jr., "Dynamic force identification based on enhanced least squares and total least-squares schemes in the frequency domain," Journal of Sound and Vibration, vol. 282, no. 1-2, pp. 37-60, 2005.
[15] C. Renzi, C. Pézerat, and J.-L. Guyader, "Local force identification on flexural plates using reduced Finite Element models," Computers \& Structures, vol. 144, pp. 75-91, 2014.

[16] X. Zhao and Y. L. Xu, "Finite element-based force identification of sliding support systems: part I-theory," Finite Elements in Analysis and Design, vol. 42, no. 4, pp. 229-248, 2006.

[17] A. S. Bahra and P. D. Greening, "Identifying multiple axial load patterns using measured vibration data," Journal of Sound and Vibration, vol. 330, no. 15, pp. 3591-3605, 2011.

[18] Y. E. Lage, N. M. M. Maia, M. M. Neves, and A. M. R. Ribeiro, "Force identification using the concept of displacement transmissibility," Journal of Sound and Vibration, vol. 332, no. 7, pp. 1674-1686, 2013.

[19] A. Berry, O. Robin, and F. Pierron, "Identification of dynamic loading on a bending plate using the Virtual Fields Method," Journal of Sound and Vibration, vol. 333, no. 26, pp. 7151-7164, 2014.

[20] P. K. Basu, A. B. Jorge, S. Badri, and J. Lin, "Higher-order modeling of continua by finite-element, boundary-element, meshless, and wavelet methods," Computers \& Mathematics with Applications, vol. 46, no. 1, pp. 15-33, 2003.

[21] B. Li and X. F. Chen, "Wavelet-based numerical analysis: a review and classification," Finite Elements in Analysis and Design, vol. 81, pp. 14-31, 2014.

[22] X. Xue, X. Zhang, B. Li, B. Qiao, and X. Chen, "Modified Hermitian cubic spline wavelet on interval finite element for wave propagation and load identification," Finite Elements in Analysis and Design, vol. 91, pp. 48-58, 2014.

[23] X. W. Zhang, X. F. Chen, and Z. J. He, "The construction of multivariable Reissner-Mindlin plate elements based on B-spline wavelet on the interval," Structural Engineering and Mechanics, vol. 38, no. 6, pp. 733-751, 2011.

[24] Z. B. Yang, X. F. Chen, X. Li, Y. Y. Jiang, H. H. Miao, and Z. J. $\mathrm{He}$, "Wave motion analysis in arch structures via wavelet finite element method," Journal of Sound and Vibration, vol. 333, no. 2, pp. 446-469, 2014.

[25] Z. Yang, X. Chen, Y. He, Z. He, and J. Zhang, “The analysis of curved beam using B-spline wavelet on interval finite element method," Shock and Vibration, vol. 2014, Article ID 738162, 9 pages, 2014.

[26] Z. Yang, X. Chen, X. Zhang, and Z. He, "Free vibration and buckling analysis of plates using B-spline wavelet on the interval Mindlin element," Applied Mathematical Modelling, vol. 37, no. 5, pp. 3449-3466, 2013.

[27] Z. Yang, X. Chen, B. Li, Z. He, and H. Miao, Vibration Analysis of Curved Shell Using B-Spline Wavelet on the Interval (BSWI) Finite Elements Method and General Shell Theory, John Wiley \& Sons, New York, NY, USA, 2012.

[28] H. Zuo, Z.-B. Yang, X.-F. Chen, Y. Xie, X.-W. Zhang, and Y. Liu, "Static, free vibration and buckling analysis of functionally graded beam via B-spline wavelet on the interval and timoshenko beam theory," Computer Modeling in Engineering \& Sciences, vol. 100, no. 6, pp. 477-506, 2014.

[29] X. Zhang, R. X. Gao, R. Yan, X. Chen, C. Sun, and Z. Yang, "Multivariable wavelet finite element-based vibration model for quantitative crack identification by using particle swarm optimization," Journal of Sound and Vibration, vol. 375, pp. 200216, 2016.

[30] H. Ronasi, H. Johansson, and F. Larsson, "Load identification for a rolling disc: finite element discretization and virtual calibration," Computational Mechanics, vol. 49, no. 2, pp. 137147, 2012. 
[31] J. Xiang, X. Chen, Y. He, and Z. He, “The construction of plane elastomechanics and Mindlin plate elements of B-spline wavelet on the interval," Finite Elements in Analysis and Design, vol. 42, no. 14-15, pp. 1269-1280, 2006.

[32] J. W. Xiang, X. F. Chen, B. Li, Z. J. He, and Y. M. He, “The construction of two-dimensional plane elasticity element using B-spline wavelet on the interval," Finite Elements in Analysis and Design, vol. 42, pp. 1268-1280, 2005.

[33] P. C. Hansen, "Analysis of discrete Ill-posed problems by means of the L-curve," SIAM Review, vol. 34, no. 4, pp. 561-580, 1992.

[34] B. Qiao, X. Zhang, C. Wang, H. Zhang, and X. Chen, "Sparse regularization for force identification using dictionaries," Journal of Sound and Vibration, vol. 368, pp. 71-86, 2016.

[35] Z. Yang, X. Chen, J. Yu, R. Liu, Z. Liu, and Z. He, "A damage identification approach for plate structures based on frequency measurements," Nondestructive Testing and Evaluation, vol. 28, no. 4, pp. 321-341, 2013.

[36] Z.-B. Yang, X.-F. Chen, Y. Xie, H.-H. Miao, J.-J. Gao, and K.-Z. Qi, "Hybrid two-step method of damage detection for plate-like structures," Structural Control and Health Monitoring, vol. 23, no. 2, pp. 267-285, 2016.

[37] J. W. Xiang, X. F. Chen, B. Li, Y. M. He, and Z. J. He, "Identification of a crack in a beam based on the finite element method of a B-spline wavelet on the interval," Journal of Sound and Vibration, vol. 296, no. 4-5, pp. 1046-1052, 2006.

[38] J. Xiang, X. Chen, Q. Mo, and Z. He, "Identification of crack in a rotor system based on wavelet finite element method," Finite Elements in Analysis and Design, vol. 43, no. 14, pp. 1068-1081, 2007. 


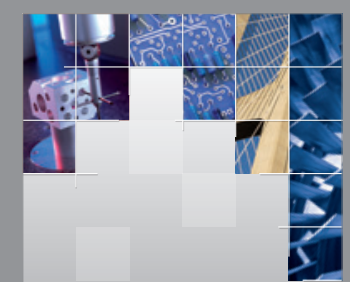

\section{Enfincering}
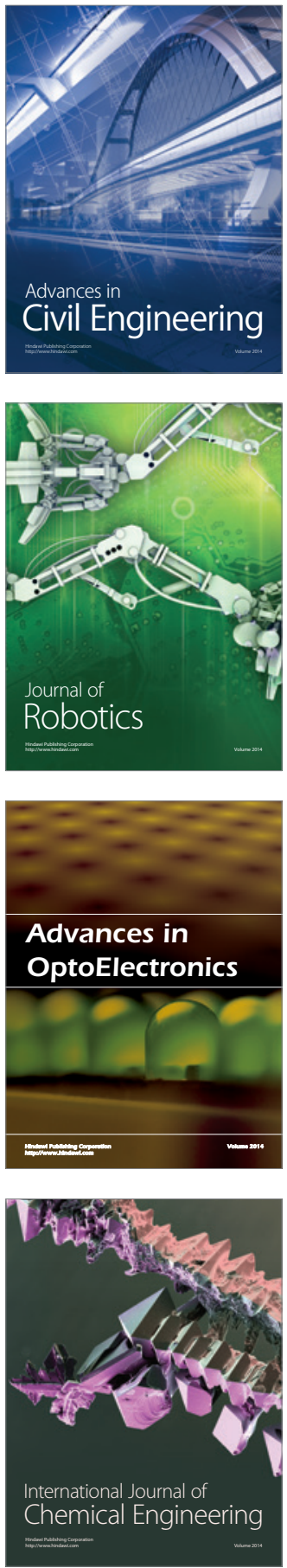

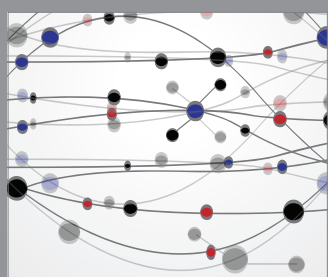

The Scientific World Journal

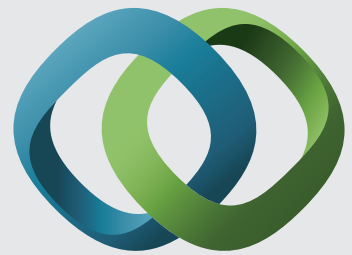

\section{Hindawi}

Submit your manuscripts at

http://www.hindawi.com
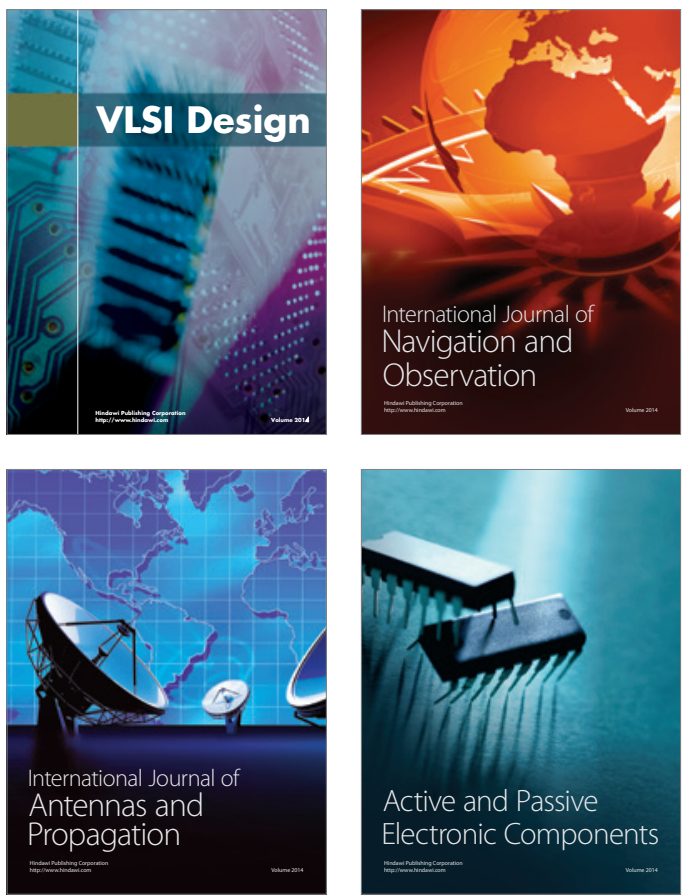


International Journal of

Distributed

Sensor Networks

Journal of

Control Science

and Engineering
